\title{
SUBSPACES OF STABILITY IN THE CAUCHY PROBLEM FOR THE HELMHOLTZ EQUATION*
}

\author{
VICTOR ISAKOV ${ }^{\dagger}$ AND STEFAN KINDERMANN ${ }^{\ddagger}$
}

\begin{abstract}
We study the stability in the Cauchy Problem for the Helmholtz equation in dependence of the wave number $k$. For simple geometries, we show analytically that this problem is getting more stable with increasing $k$. In more detail, there is a subspace of the data space on which the Cauchy Problem is well posed, and this subspace grows with larger $k$. We call this a subspace of stability. For more general geometries, we study the ill-posedness by computing the singular values of some operators associated with the corresponding well-posed (direct) boundary value problems. Numerical computations of the singular values show that an increasing subspace of stability exists for more general domains and boundary data. Assuming the existence of such subspaces we develop a theory of (Tikhonov type) regularization for the Cauchy Problem, giving convergence rates and regularization parameter choice which improve with growing $k$.
\end{abstract}

Key words. Cauchy Problem, Helmholtz equation, subspace of stability, conditional stability.

AMS subject classifications. 35R30, 35R25, 35B60, 65F15.

1. Introduction. The Cauchy Problem for the Helmholtz equation is a wellknown example of an ill-posed problem. As for other elliptic partial differential equations, its solution is unique, but does not depend continuously on the Cauchy data in standard norms $[1,9,17]$. However, the ill-posedness is more involved for the Helmholtz equation than for, e.g., the Laplace equation, because stability estimates exhibit a strong dependence on the wave number $k$. In $[25,26,14,15]$, it was shown that under certain convexity type assumptions, conditional stability for the Cauchy Problem improves with higher wave number, and in fact, turns to Lipschitz type estimates in the high frequency limit $k \rightarrow \infty$, which is an unexpected behaviour for elliptic Cauchy Problems. This effect is the main motivation for our work. We would like to investigate the transition from a severely ill-posed problem to a mildly (or even stable) problem when the wave number grows. We show analytically Lipschitz stability of the "low frequency" part of a solution, but only for simple geometries. In a more general situation, we study the problem numerically as an operator equation in Hilbert spaces. We compute the singular values of the associated forward operator. The main conclusion is that for all domains we considered, there are subspaces which are increasing with $k$, on which the Cauchy Problem is well-posed. We call these subspaces subspaces of stability. We suspect that the occurrence of these subspaces is a general property of the Cauchy Problem for the Helmholtz type equations. Under the assumption that these subspaces exist, we furthermore study the convergence of Tikhonov regularization. Theorems 1, 3, 4 state error estimates for Tikhonov regularization applied to our problem. It turns out that, similarly to the conditional stability estimates, the convergence and convergence rates show a strong dependence on the wave number (or the subspace of stability, respectively). For the limit case that the wave number $k$ tends to $\infty$, we prove - under the assumption of a growing subspace of stability - linear error rates, indicating an asymptotically well-posed problem. The

\footnotetext{
*Received October 22, 2010; accepted for publication March 31, 2011.

${ }^{\dagger}$ Department of Mathematics and Statistics, Wichita State University, Wichita, KS 67260-0033, USA (victor.isakov@wichita.edu).

$\ddagger$ Industrial Mathematics Institute, Johannes Kepler University Linz, A-4040 Linz, Austria (kindermann@indmath.uni-linz.ac.at).
} 
main contribution to a standard error analysis is the inclusion of the $k$-dependence, leading to improving rates compared to known estimates.

The paper is organized as follows: In Section 2 we state the problem in precise form, discuss some known results, and give Lipschitz stability estimates for the low frequency part of the solution to the Cauchy Problem in a square and in an annulus, when the convexity conditions in $[14,15,25]$ are violated and one has deteriorating stability of the solution for larger $k$ [17]. Moreover, we formulate this problem as an operator equation and analyze the singular values for rectangular and annular domains. In Section 3 we consider the Tikhonov regularization for this problem and prove convergence and convergence rates results. In Section 4 we look for the subspaces of stability by numerical means when analytic solutions are not available. In Section 5 we numerically computed Tikhonov regularization and give evidence on the predicted convergence rates and improving stability.

2. Problem statement and background. Let $\Omega$ be a domain with Lipschitz boundary $\partial \Omega$ and $\nu$ be the exterior unit normal to $\partial \Omega$. Let $\Gamma_{o b s}$ be a non empty open part of $\partial \Omega$, where the Cauchy data are given, and $\Gamma_{b}$ be another (open, possibly empty) part, where Neumann data are given. We are interested in the Cauchy problem for the Helmholtz equation, i.e., we want to find a solution $u$ of the problem

$$
\begin{aligned}
\left(\Delta+k^{2}\right) u & =f \quad \text { in } \Omega, \\
\left.u\right|_{\Gamma_{o b s}} & =u_{0},\left.\partial_{\nu} u\right|_{\Gamma_{o b s}}=u_{1}, \\
\left.\partial_{\nu} u\right|_{\Gamma_{b}} & =0,
\end{aligned}
$$

where $k>0$ and $u_{0}, u_{1}, f$, are given functions. We are mainly interested in the stability of the reconstruction, i.e., in the continuity of the mapping

$$
\left.\left(u_{0}, u_{1}\right) \rightarrow u\right|_{\Gamma}
$$

Here $\Gamma=\partial \Omega \backslash\left(\Gamma_{o b s} \cup \Gamma_{b}\right)$ is the remaining part of the boundary, where the unknown solution is to be reconstructed.

The Cauchy Problem for elliptic equations is a classical example of a severely illposed problem [9]. It appears in several applications such as electromagnetics [24] or acoustics [6]. Computing approximate solutions by various regularizations have been suggested by several authors, e.g., by an initial value approach [22], backpropagation [21], frequency space cut-off [24], iterative methods [19] or Tikhonov regularization $[16,27,20,28,23]$.

Analytically, the Cauchy problem for the Helmholtz equation was studied by Fritz John in his famous paper [17]. He showed that a uniform - with respect to $k$ - conditional stability estimate for (1)-(3) is at best of logarithmic type. It was observed by many researchers that the ill-posedness for this problem is more involved, because the stability of the Cauchy Problem for the Helmholtz equation has a strong dependence on the wave number, and can be increasing with $k$. This observation was put on an analytical basis in $[14,15,25,26]$. It was shown that under some geometrical (convexity type) restrictions on $\Omega$ and $\Gamma_{\text {obs }}$, conditional stability estimate yields bounds that depend on the wave number, such that for high wave number $k$ the estimates tend to Lipschitz type estimates (and hence the problem becomes a mildly ill-posed one). This is in strong contrast with John's result on logarithmic stability. Let us briefly sketch some of the results of [14].

If $\Omega$ is a domain which is the convex hull of $\Gamma_{o b s}$, and let $M$ be an a-priori bound of the $H^{1}$-norm of a solution to (1)-(3) on $\Omega$. It was shown in [14] that on a subset $\Omega_{1}$ with $\bar{\Omega}_{1} \subset \Omega \cup \Gamma_{\text {obs }}$ there exist positive constants $C, \lambda<1$ such that 


$$
\begin{aligned}
\|u\|_{(0)}\left(\Omega_{1}\right) \leq & C\left(\left\|u_{0}\right\|_{(1)}\left(\Gamma_{o b s}\right)+\left\|u_{1}\right\|_{(0)}\left(\Gamma_{o b s}\right)\right. \\
& \left.+\frac{1}{k} M^{1-\lambda}\left[k\left\|u_{0}\right\|_{(1)}\left(\Gamma_{o b s}\right)+\left\|u_{1}\right\|_{(0)}\left(\Gamma_{o b s}\right)\right]^{\lambda}\right) .
\end{aligned}
$$

Hence, the solution depends Hölder continuously on the Cauchy data and, more important, for $k \rightarrow \infty$ the solution becomes Lipschitz continuously dependent on the Cauchy data. This was named the effect of increasing stability for the large wave number $k$. Of course, (4) is in no contradiction to John's counterexample, because in [17] $\Omega$ does not intersect the convex hull of $\Gamma_{\text {obs }}$.

For linear inverse problems the degree of ill-posedness is controlled by the singular values of the forward operator [8]. The degree of ill-posedness strongly determines the convergence speed of any regularization method. Conditional stability estimates give a hint on the degree of ill-posedness, but they can only be related to this degree if the bound $M$ corresponds to the smoothness class defined by the forward operator [11]. The main aim of this work is to study the degree of ill-posedness of the Cauchy Problem for the Helmholtz equation and its dependence on the wave number $k$. Unfortunately, only for very specific domains an analytic formula for the singular values can be obtained. For more general domains we rely on a numerical computation. The main results (cf. Section 4) of this analysis are the phenomenon of a subspace of stability, i.e., a subspace of the data space on which the inverse problem can be solved in a stable way. Moreover, this region of stability grows with the wave number $k$. As an unexpected result we observed that the existence of a growing subspace of stability does not depend on the geometry of the problem.

The impact of the effect of subspaces of stability on the convergence of regularization methods is analyzed in Section 3. We find error estimates for Tikhonov regularization for problems with an increasing region of stability, where we stress the dependence on the dimension of the subspace of stability. This leads to estimates akin to (4) but in an operator theoretic formulation. Although we follow the standard error analysis (cf. [8]) using spectral theory, the subspace of stability has some important consequences, such as the appropriate use of a noise level and associated parameter choice rules. With regard to these facts we find error estimates that are asymptotically (for $k \rightarrow \infty$ ) linear in the noise level, which indicates an asymptotically well-posed problem (see Eq. (30), (31)).

In our opinion, the increasing stability in the linear Cauchy Problem (or of the continuation) has a great promise to improve resolution in many (non linear) inverse problems. Indeed, the loss of precision of the data due to continuation toward unknown objects can not be compensated. So far, for many important inverse problems, numerical resolution is very low, which severely affects useful applications.

To indicate the increased stability we first study the problem for simple geometries.

2.1. Lipschitz stability in subspaces. Now we will give stability estimates for the solution $u$ to the Cauchy Problem (1)-(3) in two simple but important cases.

EXAMPLE 1 (rectangular domain). In the first example we consider a planar rectangular domain $\Omega=(a, b) \times(0, h) \subset \mathbb{R}^{2}$, with Cauchy data on the bottom, $\Gamma_{\text {obs }}=(a, b) \times\{0\}$, homogeneous Neumann data on the left and right hand side $\Gamma_{b}=\{a, b\} \times(0, h)$, and $f=0$. A separation of variables leads to a expansion of the 
solution into a cosine series

$$
u(s, t):=\sum_{n=0}^{\infty} u_{n}(t) e_{n}(s), \quad e_{n}(s)=\cos \left(\frac{\pi n}{b-a}(s-a)\right),
$$

with $(s, t) \in(a, b) \times(0, h)$. For $\gamma \in \mathbb{N}$, we make use of the projector

$$
u(s, t ; \gamma)=\sum_{n=0}^{\gamma} u_{n}(t) e_{n}(s)
$$

to establish the following stability estimate. (We denote by $\|\cdot\|_{(0)}(\Gamma)$ the $L^{2}$-norm on a set $\Gamma$.)

LEMMA 1. Let $\gamma=\left[\frac{b-a}{\pi} k\right]$, then for any $t \in[0, h]$

$$
\left\|\partial_{t} u(., t ; \gamma)\right\|_{(0)}^{2}(a, b)+k^{2}\|u(., t ; \gamma)\|_{(0)}^{2}(a, b)-\left\|\partial_{s} u(., t ; \gamma)\right\|_{(0)}^{2}(a, b) \leq
$$

$$
\left\|u_{1}(. ; \gamma)\right\|_{(0)}^{2}\left(\Gamma_{o b s}\right)+k^{2}\left\|u_{0}(. ; \gamma)\right\|_{(0)}^{2}\left(\Gamma_{o b s}\right)-\left\|\partial_{s} u_{0}(. ; \gamma)\right\|_{(0)}^{2}\left(\Gamma_{o b s}\right) .
$$

If $u_{0}=0$, then

$$
\left\|\partial_{t} u(., t ; \gamma)\right\|_{(0)}(a, b) \leq\left\|u_{1}(. ; \gamma)\right\|_{(0)}\left(\Gamma_{o b s}\right), 0<t \leq h .
$$

If $u_{1}=0$, then

$$
\|u(., t ; \gamma)\|_{(0)}(a, b) \leq\left\|u_{0}(. ; \gamma)\right\|_{(0)}\left(\Gamma_{o b s}\right), 0<t \leq h
$$

Proof. Using (5) and (6) $u(., . ; \gamma)$ solves (1) when

$$
\partial_{t}^{2} u_{n}(t)+\omega_{n, k}^{2} u_{n}(t)=0, \quad \omega_{n, k}=\left(k^{2}-\left(\frac{\pi n}{b-a}\right)^{2}\right)^{\frac{1}{2}} .
$$

Multiplying this equality by $\partial_{t} u_{n}$ and integrating by parts yield

$$
\left(\left(\partial_{t} u_{n}\right)^{2}+\omega_{n, k}^{2} u_{n}^{2}\right)(t)=u_{1 n}^{2}+\omega_{n, k}^{2} u_{0 n}^{2} .
$$

From the definition of $u(., ; \gamma)$, the orthogonality of $e_{n}$ in $L^{2}(a, b)$, and the Parseval equality we find

$$
\begin{gathered}
\int_{a}^{b}\left(\left(\partial_{t} u\right)^{2}(s, t ; \gamma)+k^{2} u^{2}(s, t ; \gamma)-\left(\partial_{s} u\right)^{2}(s, t ; \gamma)\right) d s= \\
\int_{a}^{b}\left(u_{1}^{2}(s ; \gamma)+k^{2} u_{0}^{2}(s ; \gamma)-\left(\partial_{s} u_{0}\right)^{2}(s ; \gamma)\right) d s
\end{gathered}
$$

which establishes the bound (7).

If $u_{0}=0$, then using the Parseval equality and (8), we obtain the second claim of Lemma 1 . If $u_{1}=0$, then dividing the both sides of (8) by $\omega_{n, k}^{2}$ we similarly obtain the third claim of Lemma 1.

EXAMPLE 2 (annular domain). For the second example let $\Omega$ be the annulus $B_{R}(0) \backslash \bar{B}_{1}(0)=\left\{(s, t) \mid 1<\sqrt{s^{2}+t^{2}}<R\right\}$ with $B_{R}(x)$ the disk with 
center $x$ and radius $R$, . Let the Cauchy data be given on the inner boundary $\Gamma_{o b s}=\left\{(s, t) \mid \sqrt{s^{2}+t^{2}}=1\right\}, \Gamma_{b}$ be empty, and $f=0$. This geometry was considered by F. John in [17] to construct his famous counterexample to stability of the continuation. As before, a separation of variables in the polar coordinates leads to a Fourier series expansion of the solution in the form

$$
\begin{gathered}
u(r, \phi)=\sum_{n=0}^{\infty}\left(u_{n 1}(r) e_{n 1}(\phi)+u_{n 2}(r) e_{n 2}(\phi)\right), \\
e_{n 1}(\phi)=\cos (n \phi), \quad e_{n 2}(\phi)=\sin (n \phi) .
\end{gathered}
$$

In the following result we use the projection

$$
u(r, \phi ; \gamma)=\sum_{n=0}^{\gamma}\left(u_{n 1}(r) e_{n 1}(\phi)+u_{n 2}(r) e_{n 2}(\phi)\right) .
$$

Lemma 2. Let $\gamma=\left[\frac{k}{\sqrt{2}}\right]$ and $\Gamma=\left\{(s, t) \mid \sqrt{s^{2}+t^{2}}=R\right\}$, then

$$
\begin{gathered}
R^{-2}\left\|\partial_{\nu} u(R, . ; \gamma)\right\|_{(0)}^{2}(\Gamma)+R^{-2} k^{2}\|u(R, . ; \gamma)\|_{(0)}^{2}(\Gamma)-R^{-4}\left\|\partial_{\phi} u(R, . ; \gamma)\right\|_{(0)}(\Gamma) \leq \\
\left\|u_{1}(. ; \gamma)\right\|_{(0)}^{2}\left(\Gamma_{o b s}\right)+k^{2}\left\|u_{0}(. ; \gamma)\right\|_{(0)}^{2}\left(\Gamma_{o b s}\right)-\left\|\partial_{\phi} u_{0}(. ; \gamma)\right\|_{(0)}^{2}\left(\Gamma_{o b s}\right) .
\end{gathered}
$$

If $u_{0}=0$, then

$$
R^{-1}\left\|\partial_{\nu} u(R, . ; \gamma)\right\|_{(0)}(\Gamma) \leq\left\|u_{1}(. ; \gamma)\right\|_{(0)}\left(\Gamma_{o b s}\right) .
$$

If $u_{1}=0$, then

$$
R^{-1}\|u(R, . ; \gamma)\|_{(0)}(\Gamma) \leq\left\|u_{0}(. ; \gamma)\right\|_{(0)}\left(\Gamma_{o b s}\right)
$$

Proof. By using the Laplace operator in polar coordinates and separating variables, $u(r, \phi ; \gamma)$ solves (1) when

$$
r \partial_{r}\left(r \partial_{r} u_{n j}\right)+\left(k^{2} r^{2}-n^{2}\right) u_{n, j}=0, \quad j=1,2 .
$$

Multiplying this equality by $r^{-4} \partial_{r} u_{n j}$ and integrating by parts yield

$$
\begin{gathered}
0=\int_{1}^{r}\left(r \partial_{r}\left(r \partial_{r} u_{n j}\right)+\left(k^{2} r^{2}-n^{2}\right) u_{n j}\right) r^{-4} \partial_{r} u_{n j} d r= \\
\int_{1}^{r}\left(r^{-4} \frac{1}{2} \partial_{r}\left(r\left(\partial_{r} u_{n j}\right)\right)^{2}+\left(k^{2} r^{-2}-n^{2} r^{-4}\right) \frac{1}{2} \partial_{r} u_{n j}^{2}\right) d r= \\
\frac{1}{2}\left[r^{-2}\left(\partial_{r} u_{n j}(r)\right)^{2}+\left(k^{2} r^{-2}-n^{2} r^{-4}\right) u_{n j}^{2}(r)-\left(\partial_{r} u_{n j}\right)^{2}(1)-\left(k^{2}-n^{2}\right) u_{n j}^{2}(1)\right]+ \\
\int_{1}^{r}\left(2 r^{-3}\left(\partial_{r} u_{n j}\right)^{2}+\left(k^{2} r^{-3}-2 n^{2} r^{-5}\right) u_{n j}^{2}\right) d r .
\end{gathered}
$$


Due to our choice of $\gamma, 0 \leq k^{2} r^{-3}-2 n^{2} r^{-5}$, hence

$$
r^{-2}\left(\partial_{r} u_{n j}(r)\right)^{2}+\left(k^{2} r^{-2}-n^{2} r^{-4}\right) u_{n j}^{2}(r) \leq\left(\partial_{r} u_{n j}\right)^{2}(1)+\left(k^{2}-n^{2}\right) u_{n j}^{2}(1) .
$$

Now we can complete the proof as in Lemma 1.

Lemma 1 and Lemma 2 show that in a low frequency domain the solution depends Lipschitz continuously on the Cauchy data.

To start with the similar analysis of the ill-posedness we would like to reformulate the Cauchy Problem as an operator equation in Hilbert spaces.

2.2. Formulation as an operator equation. It is convenient to introduce the boundary value problem

$$
\begin{aligned}
\left(\Delta+k^{2}\right) v & =0 \quad \text { in } \Omega \\
\left.\partial_{\nu} v\right|_{\Gamma_{o b s}} & =0 \\
\left.\partial_{\nu} v\right|_{\Gamma_{b}} & =0 \\
\left.v\right|_{\Gamma} & =\left.u\right|_{\Gamma}
\end{aligned}
$$

Using the forward operator $A_{k}$ :

$$
A_{k}:\left.\left.v\right|_{\Gamma} \rightarrow v\right|_{\Gamma_{o b s}}
$$

where $v$ is a solution to (9)-(12), the Cauchy Problem with $u_{1}=0$ is now equivalent to the operator equation

$$
A_{k} x=y,
$$

for unknown $x=\left.v\right|_{\Gamma}$ and possibly noisy data $y=\left.v\right|_{\Gamma_{o b s}}+$ noise. The data $y$ can be obtained from the data in the Cauchy problem (1)-(3). We want to study the ill-posedness of this equation, which is indicated by the decay of the singular values of $A_{k}$. For a general domain it is hardly possible to find analytic expressions for the singular values, but for two simple examples in Section 2.1 such an expression is well known. We first look at these standard domains and later study the ill-posedness by numerical computations for other domains.

Let us first treat the Example 1 of Section 2.1. Again we assume the Cauchy data on the bottom side $\Gamma_{o b s}=(a, b) \times\{0\}$, we want to reconstruct the solution on the upper side $\Gamma=(a, b) \times\{h\}$, and we impose zero Neumann data on $\Gamma_{b}=a, b \times(0, h)$. In an operator formulation the problem implies a forward operator $A_{k}: L^{2}((a, b)) \rightarrow$ $L^{2}((a, b))$, determined by the boundary value problem (9)-(12) and (13). The operator $A_{k}$ can be calculated via the method of separation of variables, in fact if the unknown $v$ is expanded into a cosine series

$$
v=\sum_{n=0}^{\infty} x_{n} e_{n}, e_{n}(s)=\cos \left(n \frac{\pi}{b-a}(s-a)\right),
$$

a standard calculation yields

$$
v(s, t)=\sum_{n=0}^{\infty} a_{n}(t) e_{n}(s),
$$


with

$$
a_{n}(t)=\left\{\begin{array}{llc}
\frac{\cosh \left((h-t) \omega_{n, k}\right)}{\cosh \left(h \omega_{n, k}\right)} & \text { if } & k<\frac{n \pi}{b-a} \\
\frac{\cos \left((h-t) \omega_{n, k}\right)}{\cos \left(h \omega_{n, k}\right)} & \text { if } & k \geq \frac{n \pi}{(b-a)}
\end{array}\right.
$$

and

$$
\omega_{n, k}:=\sqrt{\left|k^{2}-\left(\frac{n \pi}{(b-a)}\right)^{2}\right|} .
$$

An evaluation of $a_{n}(t)$ at $t=h$ gives that $A_{k}$ is a diagonal operator. In fact, $A_{k} e_{n}=$ $d_{n}(k) e_{n}, \quad n=0,1, \ldots$, with

$$
d_{n}(k)=\left\{\begin{array}{ccc}
\frac{1}{\cos \left(h \omega_{n, k}\right)} & \text { if } \quad n \leq k \frac{(b-a)}{\pi}, \quad m=0,1, \ldots \\
\frac{1}{\cosh \left(h \omega_{n, k}\right)} & \text { if } \quad n>k \frac{(b-a)}{\pi}, \quad
\end{array}\right.
$$

The singular values of $A_{k}$ are the square roots of the eigenvalues of $A_{k}^{*} A_{k}$ :

$$
\sigma_{n}(k)=\left\{\begin{array}{cc}
\frac{1}{\left|\cos \left(h \omega_{n, k}\right)\right|} & 0 \leq n \leq k \frac{(b-a)}{\pi}, \\
\frac{1}{\cosh \left(h \omega_{n, k}\right)} & n>k \frac{(b-a)}{\pi} .
\end{array}\right.
$$

Note that we indexed the singular values starting from $n=0$ and that the values in the first line are not ordered.

REMARK. The operator $A_{k}$ is well defined for those $k$ that do not satisfy $\cos \left(h \omega_{n, k}\right)=0$ for some $n$. The roots of these equations correspond exactly to the eigenvalues of the associated boundary value problem.

The main conclusion from this example is that the singular values strongly depend on the wave number $k$. In the low frequency domain $n \leq k \frac{(b-a)}{\pi}$ the singular values of $A_{k}$ are bounded from below by 1 , while for the high frequency domain the singular values decay exponentially. The most important fact is that in the low frequency domain the operator $A_{k}$ is continuously invertible and this domain increases with $k$. This is in perfect coincidence with the stability results of Lemma 1.

We henceforth call the subspace where $A_{k}$ is continuously invertible a region of stability and the index $n$ from which the exponential decay occurs a transition point. A plot of the singular values for a rectangular domain and for different wave number is shown in the left plot Figure 5. From the analysis we see that the transition point occurs at $n \sim k \frac{(b-a)}{\pi}$, thus it is a (linear) increasing function of the wave number.

The Example 2 in Section 2.1 is another case, where the singular values are known explicitly as well. Let $\Omega$ be an annular domain $\Omega=B_{r_{1}}(0) \backslash \bar{B}_{r_{2}}(0) \subset \mathbb{R}^{2}$ with $r_{1}<r_{2}$. The Cauchy data are given on one part of the boundary $\Gamma_{o b s}=\partial B_{r_{1}}(0)$ and we want to reconstruct the solution at $\Gamma=\partial B_{r_{2}}(0)$. The solution $v$ to (9)-(12) in the polar coordinates $(r, \phi)$ is

$$
v(r, \phi)=\sum_{n=0}^{\infty}\left(C_{1 n} e_{1 n}(\phi)+C_{2 n} e_{2 n}(\phi)\right) v_{n}(r),
$$

where $v_{n}(r)$ satisfies

$$
\left.r \partial_{r}\left(r \partial_{r}\right) v_{n}(r)\right)+\left(k^{2} r^{2}-n^{2}\right) v_{n}(r)=0 .
$$


Solutions to these equations are

$$
v_{n}(r)=E_{n} J_{n}(k r)+D_{n} Y_{n}(k r),
$$

where $J, Y$ are the Bessel functions of the first and second kind. The boundary conditions for the Helmholtz problem imply the following boundary conditions on $v_{n}$

$$
\partial_{r} v_{n}\left(r_{1}\right)=0, \quad v_{n}\left(r_{2}\right)=1 .
$$

The free coefficients $E_{n}, D_{n}$ can be found from these equations yielding to

$$
\begin{aligned}
E_{n} & =\frac{-Y_{n}^{\prime}\left(k r_{1}\right)}{Y_{n}\left(k r_{2}\right) J_{n}^{\prime}\left(k r_{1}\right)-J_{n}\left(k r_{2}\right) Y_{n}^{\prime}\left(k r_{1}\right)}, \\
D_{n} & =\frac{J_{n}^{\prime}\left(k r_{1}\right)}{Y_{n}\left(k r_{2}\right) J_{n}^{\prime}\left(k r_{1}\right)-J_{n}\left(k r_{2}\right) Y_{n}^{\prime}\left(k r_{1}\right)} .
\end{aligned}
$$

It follows that $A_{k}$ is again a diagonal operator in the Fourier basis $e_{j n}$ :

$$
A_{k}\left(e_{j n}\right)=d_{n}(k) e_{j n}, \quad n=0,1, \ldots, j=1,2,
$$

with

$$
d_{n}(k)=\frac{J_{n}^{\prime}\left(k r_{1}\right) Y_{n}\left(k r_{1}\right)-J_{n}\left(k r_{1}\right) Y_{n}^{\prime}\left(k r_{1}\right)}{Y_{n}\left(k r_{2}\right) J_{n}^{\prime}\left(k r_{1}\right)-J_{n}\left(k r_{2}\right) Y_{n}^{\prime}\left(k r_{1}\right)}
$$

and the (unordered) singular values

$$
\sigma_{n}(k)=\left|d_{n}(k)\right|, n=0,1, \ldots, \text { counted with multiplicity } .
$$

Here multiplicity means that only the singular value corresponding to the constant singular function appear as single value, while all other ones are counted twice. We also note that the singular values are not ordered here, such that $\sigma_{0}(k)$ corresponding to the constant singular function is not necessarily the largest singular value.

The structure of the singular values in this example is less clear. While asymptotics of the Bessel functions are known and one can calculate their values for any particular $n$, their detailed properties (zeros, maximal values etc.) are largely unknown. The Lipschitz stability estimates of Lemma 2 suggest the behavior of singular values similar to the case of a rectangle. Indeed, from the plot of the logarithm of the ordered singular values, e.g., in Figure 4 a similar picture as for the rectangular domain can be observed: The singular values are bounded from below up to certain $n$. This transition point is again increasing with the wave number, and a subspace of stability on which $A_{k}$ is invertible increases with $k$.

3. Error estimates for Tikhonov regularization. The problem we are interested in is to find $x$ from given (possibly noisy) data $y$ for the Cauchy Problem for the Helmholtz equation in the form of the operator equation (13). In the model problem it is obvious that we are faced with an ill-posed problem, in fact, with an exponentially ill-posed problem. The most important fact in the Cauchy Problem is the increasing subspace of stability.

What we are mainly interested in here is ill-posedness and regularization for varying wave numbers and their dependence on the wave number, much like in the spirit of [14]. 
Let us recall classical Tikhonov regularization for abstract operator equations (13). A stable approximation to a solution of (13) in presence of noisy data can be found by

$$
x_{\alpha}^{\delta}=\left(A_{k}^{*} A_{k}+\alpha I\right)^{-1} A_{k}^{*} y^{\delta} .
$$

Here $y^{\delta}$ indicates noisy data, with noise level $\delta$, i.e.

$$
\left\|y-y^{\delta}\right\| \leq \delta
$$

where $y$ are exact (but unknown) data in the range of $A_{k} . \alpha>0$ is an appropriately chosen regularization parameter. The use of Tikhonov regularization is not the only choice, one can use and derive analogous error estimates for other regularizations as well.

We now study the regularization error in a general setting: Let $A_{k}$ be a familiy of compact operators mapping between Hilbert spaces $X, Y: A_{k}: X \rightarrow Y$. For an error analysis we impose the following conditions on these operators, which - we think are features of the Cauchy Problem for the Helmholtz equation.

Let $\left(\sigma_{n}(k), u_{n}^{k}, v_{n}^{k}\right)$ be the singular system of $A_{k}$, then we define the orthogonal projectors onto the stable subspaces of stability in the spaces $Y$ of solutions and $X$ of data

$$
P_{k} y:=\sum_{n=1}^{\gamma(k)}\left(y, v_{n}^{k}\right)_{Y} v_{n}^{k}, Q_{k} x:=\sum_{n=1}^{\gamma(k)}\left(x, u_{n}^{k}\right)_{X} u_{n}^{k}
$$

\section{Assumption 1.}

- $\left(A_{k}\right)_{k \in \mathcal{I}}$ is a family of compact operators between Hilbert spaces $X, Y$, indexed by real numbers $k \in \mathcal{I} \subset \mathbb{R}^{+}$.

- There exists a positive constant $\beta$ and an increasing function $\gamma(k)$ with $\lim _{k \rightarrow \infty} \gamma(k)=\infty$ such that

$$
\sigma_{n}(k) \geq \beta>0 \quad \forall n=1, \ldots, \gamma(k) .
$$

Moreover the subspaces spanned by $u_{1}^{k}, \ldots u_{n}^{k}$ are increasing with $k$ and their union over $k$ is $X$.

The following analyis is done for general family of operators $A_{k}$ satisfying Assumption 1. Of course, the Cauchy-Problem for the Helmholtz equation serves as the main example of such operators. The operators $A_{k}$ corresponding to Example 1 in Section 2.2 (Cauchy-Problem on a rectangular domain) are instances of operators satisfying Assumption 1, with the choice $X=Y=L^{2}$. In this case, if is obvious that (17) is satisfied with $\beta=1$ and $\gamma(k)=k \frac{b-a}{\pi}$. Note that $\gamma(k)$ corresponds to what we called the transition point. The operators corresponding to the annular domain are another example, where Assumption 1 holds.

The inequality (17) is central in our analysis. It states that for fixed $k$, there is a $\gamma(k)$-dimensional subspace on which $A_{k}$ can be inverted continuously. It should be noted that, as a consequence of (17), the limiting problem with operator $\lim _{k \rightarrow \infty} A_{k}$ (if it exists) will be a well-posed problem (at least on an infinite-dimensional subspace). Thus, although for fixed $k$ the problem is ill-posed we are faced here with a family if ill-posed problem converging to a well-posed one. Since all $A_{k}$ are compact, this can only happen if these operators converge pointwise but not uniformly (the uniform 
limit of compact operators is again compact). It is therefore important to keep in mind that we cannot impose an inequality (17) that is uniform in $n$, but $n$ has to depend on $k$ via $\gamma(k)$. Such a situation often arises in what Nashed classified as type-I ill-posed problems, cf. [10].

The condition that $A_{k}$ are compact is just for a simplification of the proof. It also ensures that problem (13) is ill-posed. Implicit in the assumption is the condition that $A_{k}$ are bounded, hence, there exists a constant $C(k)$ such that

$$
\sigma_{n}(k) \leq C(k) \quad \forall n \in \mathbb{N} .
$$

Again, this condition is not vital, but simplifies the analysis.

We introduce the noise level on the unstable subspace

$$
\delta_{k}:=\left\|\left(I-P_{k}\right)\left(y-y^{\delta}\right)\right\|_{Y} .
$$

We now estimate the error between the regularized solution and the true solution. The following standard notation is used: $x^{\dagger}$ is the minimum norm solution, $y$ is the exact data, and $x_{\alpha}$ is the regularized solution for exact data

$$
x_{\alpha}=\left(A_{k}^{*} A_{k}+\alpha I\right)^{-1} A_{k}^{*} y .
$$

The error between $x_{\alpha}^{\delta}$ and $x^{\dagger}$ can be bounded in the following way.

THEOREM 1. Under Assumption 1 let $\left(\sigma_{n}(k), u_{n}^{k}, v_{n}^{k}\right)$ be the singular system of $A_{k}$, then the following error estimate for Tikhonov regularization holds

$$
\begin{aligned}
\left\|x_{\alpha}^{\delta}-x^{\dagger}\right\| \leq & \alpha \frac{1}{\beta^{2}} \sqrt{\sum_{n \leq \gamma(k)}\left|\left(x_{n}^{\dagger}, u_{n}^{k}\right)_{X}\right|^{2}}+\sqrt{\sum_{n>\gamma(k)} \frac{\alpha^{2}}{\left(\alpha+\sigma_{n}(k)^{2}\right)^{2}}\left|\left(x_{n}^{\dagger}, u_{n}^{k}\right)_{X}\right|^{2}} \\
& +\frac{1}{\beta} \sqrt{\sum_{n<\gamma(k)}\left|\left(y-y^{\delta}, v_{n}^{k}\right)_{Y}\right|^{2}}+\frac{1}{\sqrt{\alpha}} \sqrt{\sum_{n>\gamma(k)}\left|\left(y-y^{\delta}, v_{n}^{k}\right)_{Y}\right|^{2}}
\end{aligned}
$$

Proof. The proof is standard (c.f. [8]) by splitting approximation error and propagated noise error and using the fact that the problem is well-posed on the stable subspace.

The previous theorem shows that the total error consists of two parts, a wellposed one corresponding to the singular values $n \leq \gamma(k)$ and an ill-posed one. Using the definition of $P_{k},(16)$ we can express the error estimate in a more concise form.

COROLlaRY 1. Under the same assumptions as in Theorem 1 we obtain the error estimate for Tikhonov regularization

$$
\begin{aligned}
\left\|x_{\alpha}^{\delta}-x^{\dagger}\right\|_{X} \leq & \alpha \frac{1}{\beta^{2}}\left\|Q_{\gamma(k)} x^{\dagger}\right\|+\left\|P_{\gamma(k)}\left(y-y^{\delta}\right)\right\| \\
& +\sqrt{\sum_{n>\gamma(k} \frac{\alpha^{2}}{\left(\alpha+\sigma_{n}(k)^{2}\right)^{2}}\left|\left(x^{\dagger}, u_{n}\right)_{X}\right|^{2}} \\
& +C(\beta) \frac{1}{\sqrt{\alpha}}\left\|\left(I-P_{\gamma}(k)\right)\left(y-y^{\delta}\right)\right\|,
\end{aligned}
$$


with constants $C\left(x^{\dagger}, \beta\right), C\left(x^{\dagger}\right)$ independent of $\alpha$ and the noise level $\delta$. Moreover, if $\alpha(\delta)$ is chosen such that $\alpha(\delta) \rightarrow 0$ and $\frac{\delta_{k}}{\sqrt{\alpha}} \rightarrow 0$, with $\delta_{k}$ as in (18) we obtain convergence

$$
\lim _{\delta \rightarrow 0}\left\|x_{\alpha}^{\delta}-x^{\dagger}\right\|_{X}=0
$$

Proof. The results can be obtained following standard proofs [8].

In the previous corollary, (19) is an estimate that is typical for well-posed problem with an error of the order $\mathcal{O}(\alpha+\delta)$. The rest, (20), (21) are estimates typical for ill-posed equations. Of course, in the asymptotics $\delta \rightarrow 0$ and $\alpha \rightarrow 0$ the ill-posed part will clearly dominate the first one, and for fixed $k$ the first line can be dropped for sufficiently small $\alpha, \delta$. However, what is most important is the fact that the ill-posed part contains only the noise level $\delta_{k}$ of the unstable subspace. Hence, as $k$ tends to $\infty,(20)$, (21) will vanish and the propagated error term does depend on $\alpha$ any more. So for large $k$ we have an almost stable problem. A standard estimate that does not include the $k$-dependence will not be able to find the correct asymptotics. To understand this in more detail we will now consider convergence rates estimates under a source condition.

3.1. Convergence rates and parameter choice. We now consider the approximation rates. It is well known, that for ill-posed problems convergence can be arbitrary bad, hence, there cannot be any convergence rates result if no additional conditions are imposed. The common way to show convergence rates is to assume an abstract smoothness condition on the exact solution $x^{\dagger}$ called source condition [8]. In a source condition one assumes that the exact solution is in the range of a certain power of the forward operator. Since in this work we are in particular interested in the asymptotic convergence with respect to the wave number $k$, it does not make sense to use a source condition, that depends on $k$. Instead we will estimate convergence rates using a source condition of a fixed reference operator $A_{0}$. This operator is assumed to be a uniform lower bound for the forward operators $A_{k}$.

To make ideas precise we use the following additional assumption:

Assumption 2. Let $\left(A_{k}\right)_{k \in \mathcal{I}}$ be a family of compact operators. We assume that there exists a compact operator $A_{0}$ and a positive constant $c_{0}$ such that for all $x \in X$ and all $k \in \mathcal{I}$

$$
c_{0}\left\|A_{0}\left(I-Q_{\gamma(k)}\right) x\right\|^{2} \leq\left\|A_{k}\left(I-Q_{\gamma(k)}\right) x\right\|^{2} .
$$

This assumption is again valid for the model problem on the rectangular domain where $A_{0}$ is the operator corresponding to $k=0$, i.e. for the Laplace equation:

LEMMA 3. Let $A_{k}$ be the forward operator for the Cauchy problem for the Helmholtz equation on a rectangular domain (cf. (14)). Moreover, let $A_{0}$ be the operator for the same problem for the Laplace equation (i.e. $k=0$ in (9)). Then Assumption 2 holds with $c_{0}=1$.

Proof. It suffices to estimate the singular values of $A_{k}$ from below by those of $A_{0}$ on the unstable domain. The singular values of $A_{k}$ in the unstable domain are given by (14) for $n>k \frac{(b-a)}{\pi}$. The singular values of $A_{0}$ can be calculated to be $\tau_{n}=\frac{1}{\left|\cosh \left(\frac{n \pi}{b-a}\right)\right|}$. 
Since $\sqrt{\left(\frac{n \pi}{b-a}\right)^{2}-k^{2}} \leq \sqrt{\left(\frac{n \pi}{b-a}\right)^{2}}$ and cosh is monotonically increasing we immediately get

$$
\cosh \left(\sqrt{\left(\frac{n \pi}{b-a}\right)^{2}-k^{2}}\right) \leq \cosh \left(\sqrt{\left(\frac{n \pi}{b-a}\right)^{2}}\right)
$$

hence

$$
\tau_{n} \leq \sigma_{n}(k)
$$

which proves the lemma. $\square$

We continue with the error analysis for Tikhonov regularization. We impose a source condition of Hölder type on $x^{\dagger}$ with respect to $A_{0}$, i.e. we assume that for some $\mu \in(0,1]$ there exists a $w \in X$ such that

$$
x^{\dagger}=\left(A^{*} A\right)^{\mu} w,
$$

or in other words if $\tau_{n}$ are the singular values of $A_{0}$ then we assume that

$$
\sum_{n=1}^{\infty} \frac{1}{\tau_{n}^{2 \mu}}\left|\left(x^{\dagger}, u_{n}\right)_{X}\right|^{2}=\|w\|<\infty .
$$

We can estimate the term in (20) in the following way

Proposition 1. Under Assumptions 1, 2, if $x^{\dagger}$ satisfies (23) with $\mu \leq 1$, the following bound holds

$$
\sum_{n>\gamma(k)} \frac{\alpha^{2}}{\left(\alpha+\sigma_{n}(k)^{2}\right)^{2}}\left|\left(x^{\dagger}, u_{n}\right)_{X}\right|^{2} \leq C\left(\mu, c_{0}\right) \alpha^{2 \mu}\left\|\left(I-Q_{\gamma(k)}\right) w\right\|^{2} .
$$

Proof. From (22) it follows that $\sigma_{n}(k)$, the singular values of $A_{k}$ and $\tau_{n}$, the singular values of $A_{0}$ satisfy

$$
\sigma_{n}(k)^{2} \geq c_{0} \tau_{n}^{2} \quad \forall n>\gamma(k) .
$$

This, together with (23) immediately yields

$$
\begin{aligned}
& \sum_{n>\gamma(k)} \frac{\alpha^{2}}{\left(\alpha+\sigma_{n}(k)^{2}\right)^{2}}\left|\left(x^{\dagger}, u_{n}\right)_{X}\right|^{2} \leq \sum_{n>\gamma(k)} \frac{\alpha^{2}}{\left(\alpha+c_{0} \tau_{n}^{2}\right)^{2}}\left|\left(x^{\dagger}, u_{n}\right)_{X}\right|^{2} \\
& \leq C\left(\mu, c_{0}\right) \alpha^{2 \mu} \sum_{n \geq \gamma(k)} \frac{1}{\tau_{n}^{2 \mu}}\left|\left(x^{\dagger}, u_{n}\right)\right|^{2}=C\left(\mu, c_{0}\right) \alpha^{2 \mu}\left\|\left(I-Q_{\gamma(k)}\right) w\right\|^{2}
\end{aligned}
$$

The Hölder source condition (23) is not the most appropriate for exponentially ill-posed problem. A logarithmic source condition has the form for some $p>0$

$$
x^{\dagger}=\log ^{-p}\left(\frac{e\left\|A_{0}\right\|^{2}}{A_{0}^{*} A_{0}}\right) w .
$$

Such condition were firstly used in [12]; for the simple case of a rectangular domain this can be rephrased as a smoothness condition that the exact solution is in some Sobolev space $H^{p^{\prime}}$ where $p^{\prime}$ is related to $p$ in (24) 
The estimate for a logarithmic source condition takes the form

Proposition 2. Under Assumptions 1, 2, if $x^{\dagger}$ satisfies (24), the following bound holds for $0<\alpha \leq \alpha_{0}$ with fixed $\alpha_{0}$

$$
\sum_{n>\gamma(k)} \frac{\alpha^{2}}{\left(\alpha+\sigma_{n}(k)^{2}\right)^{2}}\left|\left(x^{\dagger}, u_{n}\right)_{X}\right|^{2} \leq C\left(p, c_{0}\right) \log ^{-2 p}\left(\frac{e \alpha_{0}}{\alpha}\right)\left\|\left(I-Q_{\gamma(k)}\right) w\right\|^{2} .
$$

Proof. The proof is similar to that in Proposition 1, where instead of $\alpha^{\mu}$ the corresponding function $\log ^{-p}\left(\frac{e\left\|A_{0}\right\|^{2}}{\alpha}\right)$ is used, together with the bound in $[12$, Eq. (2.1)] and (24) and the fact that that $\left(I-Q_{\gamma(k)}\right)$ commutes with $A_{0}^{*} A_{0}$.

Collecting the results from Corollary 1 and Proposition 1 and we come to the main estimates.

ThEOREM 2. Let Assumptions 1 and 2 hold. If $x^{\dagger}$ satisfies a source condition (23), then the error for Tikhonov regularization can be bounded by

$$
\begin{aligned}
& \left\|x_{\alpha}^{\delta}-x^{\dagger}\right\| \leq C_{1} \alpha\left\|Q_{\gamma(k)} x^{\dagger}\right\|+C_{2}\left\|P_{\gamma(k)}\left(y-y^{\delta}\right)\right\| \\
& +C_{3} \alpha^{\mu}\left\|\left(I-Q_{\gamma(k)}\right) w\right\|+C_{4} \frac{1}{\sqrt{\alpha}} \|\left(I-P_{\gamma(k)}\left(y-y^{\delta}\right) \|,\right.
\end{aligned}
$$

where $C_{1}, C_{2}, C_{3}, C_{4}$ are constants independent of $\alpha, \delta$. If $x^{\dagger}$ satisfies a logarithmic source condition (24) then the following bound holds

$$
\begin{aligned}
& \left\|x_{\alpha}^{\delta}-x^{\dagger}\right\| \leq C_{1} \alpha\left\|Q_{\gamma(k)} x^{\dagger}\right\|+C_{2}\left\|P_{\gamma(k)}\left(y-y^{\delta}\right)\right\| \\
& +C_{3} \log ^{-p}\left(\frac{e \alpha_{0}}{\alpha}\right)\left\|\left(I-Q_{\gamma(k)}\right) w\right\|+C_{4} \frac{1}{\sqrt{\alpha}} \|\left(I-P_{\gamma(k)}\left(y-y^{\delta}\right) \| .\right.
\end{aligned}
$$

The main point in this theorem is the fact that $\lim _{k \rightarrow \infty}\left\|\left(I-Q_{\gamma(k)}\right) w\right\|=0$ and $\lim _{k \rightarrow \infty}\left\|\left(I-P_{\gamma(k)}\right)\left(y-y^{\delta}\right)\right\|=0$. Hence, for high wave numbers the terms in $(21)$, (26) and (28) vanish; since they correspond to the ill-posedness we see that as $k \rightarrow \infty$ we obtain estimates for a well-posed problem.

The estimates in Theorem 2 depend on $\alpha$; for a convergence rate estimate one has to specify a parameter choice rule $\alpha(\delta)$. Note that for Hölder source condition (23) a well-known a-priori parameter choice rule

$$
\alpha=\delta^{\frac{2}{2 \mu+1}}
$$

gives optimal order results (cf., e.g., [8]). However, such a choice does not take into account the $k$-dependence of the constants. In fact, if we plug in (29) into (25), (26) we obtain

$$
\left\|x_{\alpha}^{\delta}-x^{\dagger}\right\| \sim \delta^{\frac{2}{2 \mu+1}}+\delta+o(k)\left(\delta^{\frac{2}{2 \mu+1}}\right)+\delta_{k} \delta^{-\frac{2}{2 \mu+1}} .
$$

For a fixed $k$ these are optimal rates. However, for $k \rightarrow \infty$ we see that the estimates are of order $\delta^{\frac{2}{2 \mu+1}}$. However, if we first let $k \rightarrow \infty$ we have gotten an error of order $\mathcal{O}(\alpha+\delta)$, if we choose now $\alpha \sim \delta$ we obtain linear rates of order $\delta$. This indicates that a parameter choice independent of $k$ does not yield the best possible asymptotic rates for large $k$ (although it does for fixed $k$ ). Instead one should use a parameter choice rule that depends on $k$ and in particular on the projected noise level $\delta_{k}$. Such 
a strategy turns out to be successful. For a analysis of this idea we need the following lemma.

Lemma 4. Let $s, \tau>0, \mu \in(0,1]$ and define

$$
f(\alpha)=\alpha+\alpha^{\mu} s+\frac{\tau}{\sqrt{\alpha}}
$$

Then,

$$
\min _{\alpha} f(\alpha) \geq C\left(\tau^{\frac{2}{3}}+\tau^{\frac{2 \mu}{2 \mu+1}} s^{\frac{1}{2 \mu+1}}\right) .
$$

Moreover, with

$$
\alpha_{*}:=\min \left\{\tau^{\frac{2}{3}},\left(\frac{\tau}{s}\right)^{\frac{2}{2 \mu+1}}\right\}
$$

we find that

$$
f\left(\alpha_{*}\right) \leq C^{\prime}\left(\tau^{\frac{2}{3}}+\tau^{\frac{2 \mu}{2 \mu+1}} s^{\frac{1}{2 \mu+1}}\right)
$$

with constants $C, C^{\prime}$ independent of $s, \tau$.

Proof. With $\xi=\frac{\alpha}{\tau^{\frac{2}{3}}}, \zeta=\frac{\alpha s^{\frac{2}{2 \mu+1}}}{\tau^{\frac{2}{2 \mu+1}}}$ we obtain the lower bound on $f(\alpha)$ :

$$
f(\alpha)=\tau^{\frac{2}{3}} \xi+\frac{1}{2 \sqrt{\xi}}+\tau^{\frac{2 \mu}{2 \mu+1}} s^{\frac{1}{2 \mu+1}}\left(\zeta^{\mu}+\frac{1}{2 \sqrt{\xi}}\right) \geq C_{1} \tau^{\frac{2}{3}}+C_{2} \tau^{\frac{2 \mu}{2 \mu+1}} s^{\frac{1}{2 \mu+1}}
$$

Here we used that $c_{1} x^{\mu}+c_{2} \frac{1}{\sqrt{x}}$ is bounded from below for all $x$ and for $\mu \in(0,1]$. Now to the upper bound for the given choice of $\alpha_{*}$ : First suppose that $\alpha_{*}=\tau^{\frac{2}{3}}$, which is the case when

$$
\tau^{\frac{2}{3}} \leq\left(\frac{\tau}{s}\right)^{\frac{2}{2 \mu+1}}
$$

This implies

$$
\begin{aligned}
s & \leq \tau^{\frac{2(1-\mu)}{3}} \\
\alpha_{*}^{\mu} s & =\tau^{\frac{2 \mu}{3}} s \leq \tau^{\frac{2 \mu}{3}} \tau^{\frac{2(1-\mu)}{3}}=\tau^{\frac{2}{3}}=\alpha^{*} .
\end{aligned}
$$

Thus, $f\left(\alpha^{*}\right) \leq 3 \tau^{\frac{2}{3}}$. The other case $\alpha_{*}=\left(\frac{\tau}{s}\right)^{\frac{2}{2 \mu+1}}$ implies

$$
\begin{aligned}
\tau^{\frac{2(1-\mu)}{3}} & \leq s \\
\tau^{\frac{2}{3}} & \leq \tau^{\frac{2}{3}} \leq \tau^{\frac{2 \mu}{3}} s \leq 2 \tau^{\frac{2}{3}}+\tau^{\frac{2 \mu}{3}} \tau^{\frac{2(1-\mu)}{3}} \leq 3 \tau^{\frac{2}{3}} .
\end{aligned}
$$

Similarly, we see that $\alpha_{*} \leq \alpha_{*}^{\mu} s$, thus, we get in the second case

$$
f\left(\alpha_{*}\right) \leq 2 \alpha_{*}^{\mu} s+\frac{\tau}{\sqrt{\alpha_{*}}}=3 \tau^{\frac{2 \mu}{2 \mu+1}} s^{\frac{1}{2 \mu+1}} .
$$

In any case the bound of the lemma holds with $C^{\prime}=3$. 
This lemma can immediately be be applied to the right hand side of (25), (26) with $s=C_{3}\left\|\left(I-Q_{\gamma(k)}\right) w\right\|$ and $\tau=C_{4} \|\left(I-P_{\gamma(k)}\left(y-y^{\delta}\right) \|\right.$, which yields the following desired convergence rates result.

ThEOREM 3. Let Assumptions 1 and 2 hold. If $x^{\dagger}$ satisfies a source condition (23) and $\alpha$ is chosen as

$$
\alpha:=\min \left\{\delta_{k}^{\frac{2}{3}},\left(\frac{\delta_{k}}{\left\|\left(I-Q_{\gamma(k)}\right) w\right\|}\right)^{\frac{2}{2 \mu+1}}\right\}
$$

with $\delta_{k}$ as in (18), then we have the error estimate for Tikhonov regularization

$$
\left\|x_{\alpha}^{\delta}-x^{\dagger}\right\| \leq C\left(\delta+\delta_{k}^{\frac{2}{3}}+\delta_{k}^{\frac{2 \mu}{2 \mu+1}}\left\|\left(I-Q_{\gamma(k)}\right) w\right\|^{\frac{1}{2 \mu+1}}\right) .
$$

This theorem is interesting for several reasons: It is clear that for fixed $k$ the domainting term in (30) is of order $\delta_{k}^{\frac{2 \mu}{2 \mu+1}}$, which is exactly the optimal order rate to be expected. However, it should be noted that this term comes with a factor that tends to 0 as $k \rightarrow \infty$. Hence it could be possible that $\delta_{k}^{\frac{2}{3}}$ is the largest term for large $k$. Moreover, in the limit as $k \rightarrow \infty$, all terms involving $\delta_{k}$ vanish, thus yielding estimates that are linear in $\delta$, which is typical for well-posed problems. This shows that including the $k$-dependence in the estimates and in the parameter choice rule we find the expected asymptotic linear estimates. An important advice is that the regularization parameter has to be chosen not depending on the actual noise level, but on the projected noise level $\delta_{k}$. But it should be noted that a naive choice driven by analogy to the standard ill-posed case like $\alpha=\delta_{k}^{\frac{2}{2 \mu+1}}$ leads to an estimate of order $\delta+\delta_{k}^{\frac{2}{2 \mu+1}}+o(k) \delta_{k}^{\frac{2 \mu}{2 \mu+1}}$. Although this are optimal rates with respect to $\delta$, the second term is suboptimal compared to the $\delta_{k}^{\frac{2}{3}}$ rates in (30).

For the case of a logarithmic source condition a similar estimate holds. We first state the analogue to Lemma 4.

Lemma 5. Let $s>0,0<\tau<\tau_{\max }, p \in \mathbb{R}^{+}$and define $\phi_{p}(\lambda):=\log ^{-p}\left(\frac{c_{0}}{\lambda}\right)$, for all $\lambda \leq c_{1}<c_{0}$. For the function

$$
f(\alpha)=\alpha+\phi_{p}(\alpha) s+\frac{\tau}{\sqrt{\alpha}}
$$

we find with the choice

$$
\alpha_{*}:=\min \left\{\tau^{\frac{2}{3}}, \frac{\tau}{s}, c_{1}\right\}
$$

that

$$
f\left(\alpha_{*}\right) \leq C^{\prime}\left(\tau^{\frac{2}{3}}+s \phi_{p}\left(\min \left\{\frac{\tau}{s}, c_{1}\right\}\right)\right) \quad \forall s>0 \text { and } 0<\tau<\tau_{\max }
$$

with a constant $C^{\prime}$ depending on $c_{1}, p, \tau_{\max }$, but not on $s, \tau$.

Proof. If $\alpha_{*}=c_{1}$, then $c_{1} \leq \tau^{\frac{2}{3}}$, hence

$$
f\left(\alpha_{*}\right)=c_{1}+\frac{\tau}{\sqrt{c_{1}}}+s \phi_{p}\left(c_{1}\right) \leq C\left(\tau_{\max }, c_{1}\right) \tau^{\frac{2}{3}}+s \phi_{p}\left(\min \left\{\frac{\tau}{s}, c_{1}\right\}\right)
$$


Let us now assume that $\alpha_{*}=\tau^{\frac{2}{3}}$, i.e. $\tau^{\frac{2}{3}} \leq \min \left\{\frac{\tau}{s}, c_{1}\right\}$. Then with the monotonicity of $\phi_{p}$

$$
f\left(\alpha^{*}\right)=\tau^{\frac{2}{3}}+\frac{\tau}{\tau^{\frac{1}{3}}}+s \phi_{p}\left(\tau^{\frac{2}{3}}\right) \leq 2 \tau^{\frac{2}{3}}+s \phi_{p}\left(\min \left\{\frac{\tau}{s}, c_{1}\right\}\right),
$$

thus the estimate in the lemma holds in this case with $C^{\prime}=2$. In the other case $\alpha_{*}=\frac{\tau}{s}$ we obtain in a similar manner $\frac{\tau}{s} \leq \tau^{\frac{2}{3}}$, and $\frac{\tau}{s} \leq c_{1}$, hence,

$$
f\left(\alpha^{*}\right)=\alpha^{*}+s\left(\phi_{p}\left(\frac{\tau}{s}\right)+\sqrt{\frac{\tau}{s}}\right) \leq \tau^{\frac{2}{3}}+s C_{2} \phi_{p}\left(\frac{\tau}{s}\right)=\tau^{\frac{2}{3}}+s C_{2} \phi_{p}\left(\min \left\{\frac{\tau}{s}, c_{1}\right\}\right) .
$$

where we used that $\phi_{p}(x)+\sqrt{x} \leq C_{2} \phi_{p}(x)$ for $x \leq c_{1}$ (cf. [13, Thm 5]).

As before, we obtain convergence rates under logarithmic source conditions.

Theorem 4. Let Assumptions 1 and 2 hold. If $x^{\dagger}$ satisfies a source condition (24) with some $p>0$ and $\alpha$ is chosen as

$$
\alpha:=\min \left\{\delta_{k}^{\frac{2}{3}},\left(\frac{\delta_{k}}{\left\|\left(I-Q_{\gamma(k)}\right) w\right\|}\right), \frac{e}{2}\left\|A_{0}\right\|^{2}\right\}
$$

with $\delta_{k}$ as in (18), then we have the error estimate for the Tikhonov regularization

$$
\left\|x_{\alpha}^{\delta}-x^{\dagger}\right\| \leq C\left(\delta+\delta_{k}^{\frac{2}{3}}+\left\|\left(I-Q_{\gamma(k)}\right) w\right\| \log ^{-p}\left(\frac{e\left\|A_{0}\right\|^{2}}{\min \left\{\frac{e\left\|A_{0}\right\|^{2}}{2}, \frac{\delta_{k}}{\left\|\left(I-Q_{\gamma(k)}\right) w\right\|}\right\}}\right)\right) .
$$

It can be observed that the structure of this estimate is similar as in the Hölder case. Again, asymptotically as $k \rightarrow \infty$ we obtain linear rates in $\delta$.

Another important observation is that the noise level for such problems, is the projection of the usual noise $\delta$ to the unstable subspace. Choosing the regularization parameter depending on $\delta$ again does not yield the asymptotically optimal rate. Instead, the effective noise level $\delta_{k}$ should be used.

It might be quite difficult to find this noise level in practice, because not only $\delta$ has to be known but also the unstable subspace. For problems, where the singular value expansion is not analytically computable this might be a serious problem. It should be mentioned, that a noiselevel-free (or heuristic ) parameter choice rule can be useful here. A recent result [18], shows, however, that under certain (not too restrictive) conditions on the noise, the quasi-optimality principle for Tikhonov regularization will converge with the optimal rate.

4. Numerical analysis of regions of stability. For the case of a rectangular domain, we found that the stable subspaces are increasing with $k$. In the case of an arbitrary domain an analytical solution cannot be found and it is not clear that a similar behaviour of the singular values holds for an arbitrary domain. We therefore analyzed the problem numerically and solved the Helmholtz equation by finite elements and computed the associated singular values. Using finite elements, the forward operator $v \rightarrow u$ can be discretized in a straightforward manner, which leads to a discrete operator

$$
A_{d i s c}: \sum_{i} x_{h, i} \phi_{i}(x) \rightarrow \sum_{i} y_{h, i} \phi_{i}(x)
$$


where $\phi_{i}$ are the corresponding finite element basis function. The discretized operator can be represented by a matrix that maps the vector $x_{h, i}$ to $y_{h, i}$

$$
A_{h}\left(x_{h, i}\right)_{i}=\left(y_{h, i}\right)_{i}
$$

Since we want to approximate the continuous problem we have to equip the finite dimensional vector spaces with a norm. Since $A$ is defined on $L^{2}$ spaces, the corresponding finite dimensional spaces are

$$
\left\|\left(x_{h, i}\right)_{i}\right\|^{2}:=\left(x_{h, i}\right)_{i}^{T} M_{x, h}\left(x_{h, i}\right)_{i}, \quad \text { and }\left\|\left(y_{h, i}\right)_{i}\right\|^{2}:=\left(y_{h, i}\right)_{i}^{T} M_{y, h}\left(y_{h, i}\right)_{i},
$$

here $M_{x, h}$ and $M_{y, h}$ are the boundary mass matrices defined by the finite element basis function:

$$
\left(M_{x, h}\right)_{i, j}=\int_{\Gamma} \phi_{i}(x) \phi_{j}(x) d \sigma(x) \quad\left(M_{y, h}\right)_{i, j}=\int_{\Gamma_{o b s}} \phi_{i}(x) \phi_{j}(x) d \sigma(x),
$$

where $\Gamma_{o b s}$ is the part of the boundary where the data are given and $\Gamma$ is the part with the unknown Dirichlet data. The approximation of the singular values of $A$ is found by a computation of the singular values of the discretize matrix

$$
\sigma_{h}=S V D\left(M_{y, h}^{\frac{1}{2}} A_{h} M_{x, h}^{-\frac{1}{2}}\right)
$$

The square roots of the mass matrix can in fact be replaced by triangular matrices coming from a Cholesky decomposition of the mass matrices. All the computation of the Cholesky factors and the singular values used the corresponding matlab routines.

For the Helmholtz equation it is well-known, that a standard finite element approximation does not give a uniform approximation independent of $k$, (this is called the pollution effect in [2]). For a fixed $k$ the finite element method does converge, though, as the grid size tends to 0 .

In a first computation we tried to get an idea of the discretization error involved in the FEM computations. For this we calculated the singular values for the operator $A_{k}$ corresponding to a rectangular domain $[0,1] \times[0, \pi]$, for which the singular values are known analytically by (14). The first Figure 1 and Figure 2 show a comparison of the numerically computed singular values using a discretization of the operator $A_{k}$ on a FEM grid with standard piecewise linear triangular elements with a total of 13281 nodes. In Figure 1 we plotted the logarithm of the numerically computed singular values (full line) as explained before and the logarithm of the exact one (dashed) for four different wave numbers $k=5.1,15.1,40.1,77.6$. (The graphs on the left hand side correspond to the lower wave numbers). It can be observed, that for the low wave numbers $k=5.1, k=15.1$ (left graphs) there is an almost perfect match between computed and exact solution. However, for larger values of $k=40.1,77.6$ there is a systematic error: the difference is larger for large singular values, which can be explained, because in the situation when the first singular values are large, the wave number is close to an eigenvalue, hence, some problems in the approximation could be expected. We further observed, that the transition from stable to unstable subspace (this is the point where the graphs have a kink) is slightly underestimated for the numerically computed values. In fact the numerical values show an earlier transition to instability. Moreover, this error in the transition grows as the the wave number grows. This corresponds to the increasing gap between the full and the dashed line at the right hand side of the plot. An interpretation of this behavior is that for 


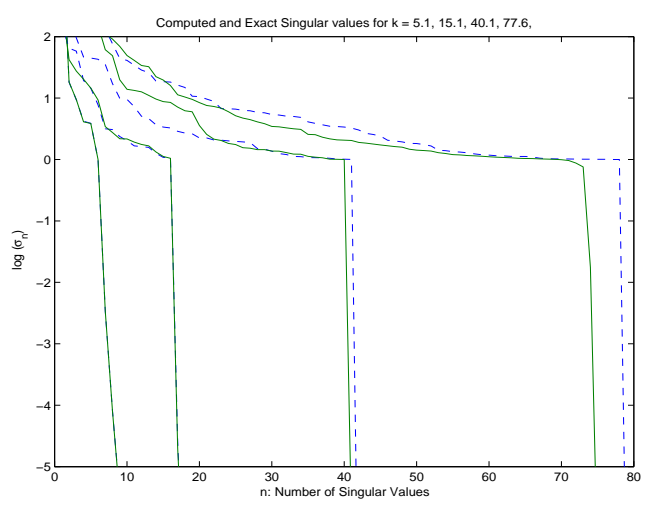

FIG. 1. Numerically computed singular values and exact singular values (dashed '-') for different wave numbers $k$ for the rectangular domain

larger $k$ the finite element computation becomes less accurate. Moreover, because the transition point for larger $k$ takes place at the higher singular values, we have to take into account that the computation of the larger singular values is in general less accurate.

What we want to point out that the numerically computed picture of the singular values does give indeed a qualitative picture of the singular values. We are most interested in the computation of the transition point and the numerical evidence that the transition point is increasing with the wave number, which was the main feature of the whole problem. Although it should be kept in mind that the numerical transition point is slightly underestimated for large wave numbers $k$.

Figure 2 gives a two-dimensional plot of the logarithm of the absolute error of the logarithm of the singular values $\operatorname{err}=\left|\log \left(\sigma_{n}(k)\right)-\log \left(\sigma_{n}^{\text {comp }}(k)\right)\right|$ plotted against $n$ the index number of the singular values and $k$. The picture again indicates where the numerical approximation is less accurate. For small singular values in the situation when $k$ is close to an eigenvalue and at the transition point. The latter inaccuracy can be seen by the 'ridge' that appears in the middle of the picture. At the rest there is quite good approximation. Since we are mainly interested in the transition point and the inverse problem, the inaccuracies of the large singular values are not so important, as they do not contribute to the ill-posedness. The inaccuracy of estimating the right transition point should be taken into account; we therefore cannot assume that the exact cut-off value for the projector $P_{k}$ is known. Still the qualitative behavior of the singular values (a region of stability that grows with $k$ ) is appearant in the numerical computation as well.

We did the same comparison for the case of an annulus domain, where an explicit solution of the singular values is known as well (compare (15)), where the observation was given at the outer radius $r_{1}=1$ and the unknown was taken at the inner radius of the annulus at $r_{2}=0.4$. Here, it has to be taken into account that the computed singular values (15) correspond to the trigonometric function on $[0, \pi]$, i.e., the singular values on the boundaries are considered to be parametrized by arclength (which corresponds to the space $L^{2}([0,2 \pi]$. In order to compare with the numerical solution, however, one has to take the inner product with respect to the surface measure, i.e. $L^{2}\left(\left[0,2 \pi r_{1}\right]\right)$ and $L^{2}\left(\left[0,2 \pi r_{2}\right]\right)$. For a circle the only difference in these norms is a constant factor of $r_{1}, r_{2}$, respectively. Similar as in (32) we therefore compared the 


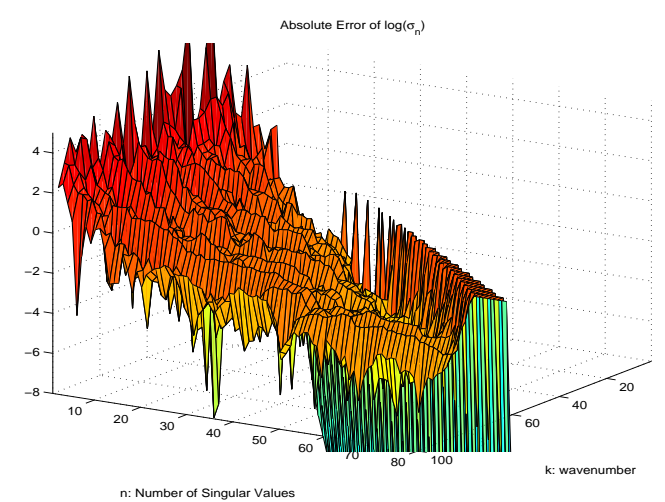

FIG. 2. Absolute Error $\left|\log \sigma_{n}^{k}-\log \sigma_{d i s c, n}^{k}\right|$ for the rectangular domain

computed singular values with that of (15) multiplied with a compensation factor $\frac{\sqrt{r_{1}}}{\sqrt{r_{2}}}$.

Figures 3 and 4 show a comparison of the computed singular values with the theoretical ones, similar as in Figure 1 and Figure 2, respectively. The results are similar as in the rectangular case: For the small wave number and away from the eigenvalues we obtain a good agreement, while for larger wave numbers and at large singular values the approximation is deteriorating.

The main conclusion from these examples is that a numerical computation gives a qualitative picture of the behavior of the singular values, which, however, might be less accurate for large wave numbers. As a rough estimate we think that with a moderate size computation grid we can get a good estimate for wave numbers up to $k \sim 50$.

The rectangular domain is one of the few examples, where the singular values can be calculated explicitly. For general domains we have to rely on the numerical computation.

In order to take the discretization into account we computed for a given domain the associated singular values of $A_{k, h}$ for several different increasingly finer grids. If the results for two successive, sufficiently small grid sizes are close to each other, the computed singular values are supposed to be close to the true ones. In this way we could estimate for what values of $k$ we could trust the numerical computations. It turns out, that the error between two successive grids does depend on $k$. What we are most interested in is to find the subspace of stability, i.e. where $\sigma_{n} \geq 1$.

In Figures 5-10 we calculated the singular values for several different domains, and different configurations of observation and unknown part of the boundary. In the left pictures the domain is indicated as well as the part of the boundary, where data are given (observation part: green boundary) and the part where, the unknown solution is sought (unknown part: red boundary). The pictures on the right hand side show a plot of the logarithm of the computed singular values for four different wave numbers $k=5,15,40,80$. There solid thick lines correspond to the finest discretization, while the thin dashed line correspond to a coarser discretization. It can be seen that the difference between two discretization is small as long as $k \leq 40$. From the pictures it can be seen that there is a transition from a stable subspace to an unstable subspace, which occurs around $\log \left(\sigma_{n}\right) \sim 0$ and is indicated by a more or less pronounced kink in the plots. It is obvious that the point of transition is increasing with the wave 


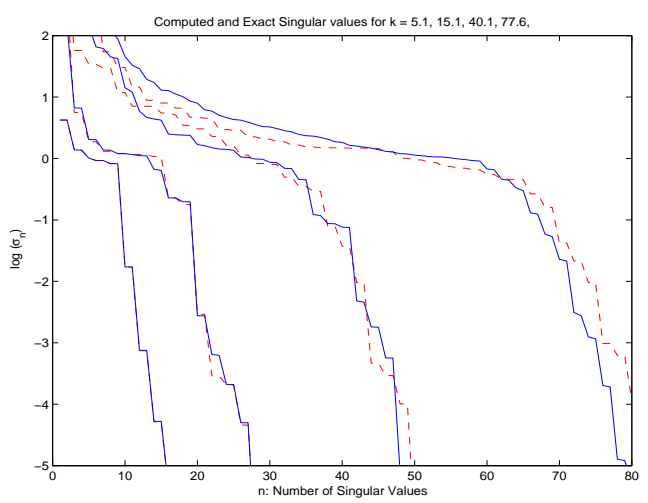

FIG. 3. Numerically computed singular values and exact singular values (dashed '-') for different wave numbers $k$ for the annular domain

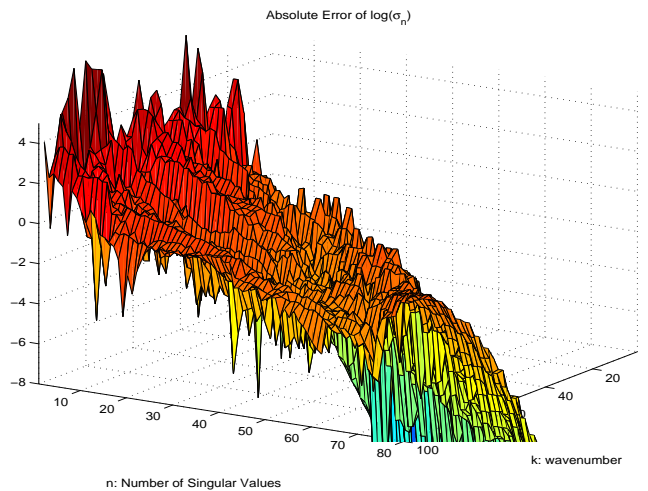

FIG. 4. Absolute Error $\left|\log \sigma_{n}^{k}-\log \sigma_{\text {disc, } n}^{k}\right|$ for the annular domain

number. The slope of the line beyond the transition point indicates the degree of ill-posedness. It can be seen that this certainly depends on the geometry. Note that the decay of the singular values is similar for similar geometries with observation and unknown part switched. Out of these figure it seems that the annulus case has a very slow decay of the singular values. (Note that the $\mathrm{x}$-axis is scaled differently in Figures $8,9,10)$. In the annulus case one can observe a staircasing, which comes from the double eigenvalues corresponding to sin and cos. The same is true for the multiple inclusion domain Figure 10, that was motivated by the paper [5].

In Figures 11-13 we tried to indicate the subspace of stability. The solid line in the pictures corresponds to an interpolated curve to those values where $\log \left(\sigma_{n}(k)\right)=1$, i.e. where $\sigma_{n}(k)=1$. The dashed lines in the plot correspond to the same curve but on a coarse discretization. It can be observed, that at low wave number (up to $k \sim 30$ they are almost identical, which indicates that our numerical approximation is accurate. The region below the solid line correspond to the subspace of stability, i.e. for a fixed wave number there is an increasing number of singular values that are bounded from below by 1 . Note that the choice of the zero level curve is rather arbitrary, we could as well have chosen a fixed positive constant. We choose 0 because the Figures 5-9 indicate a kink there. It is obvious that the region of stability is increasing with the 

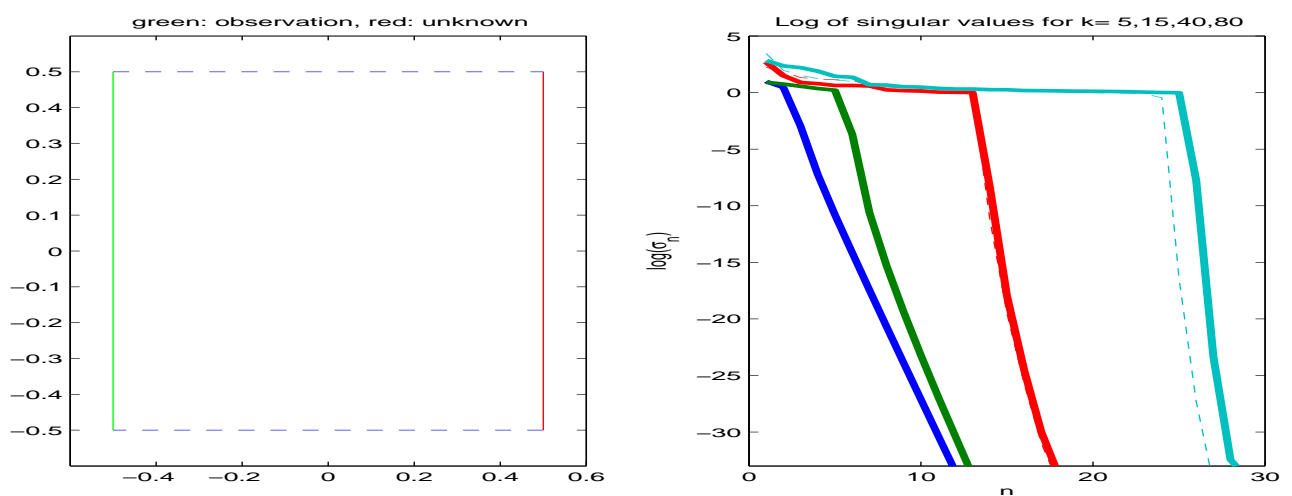

FIG. 5.
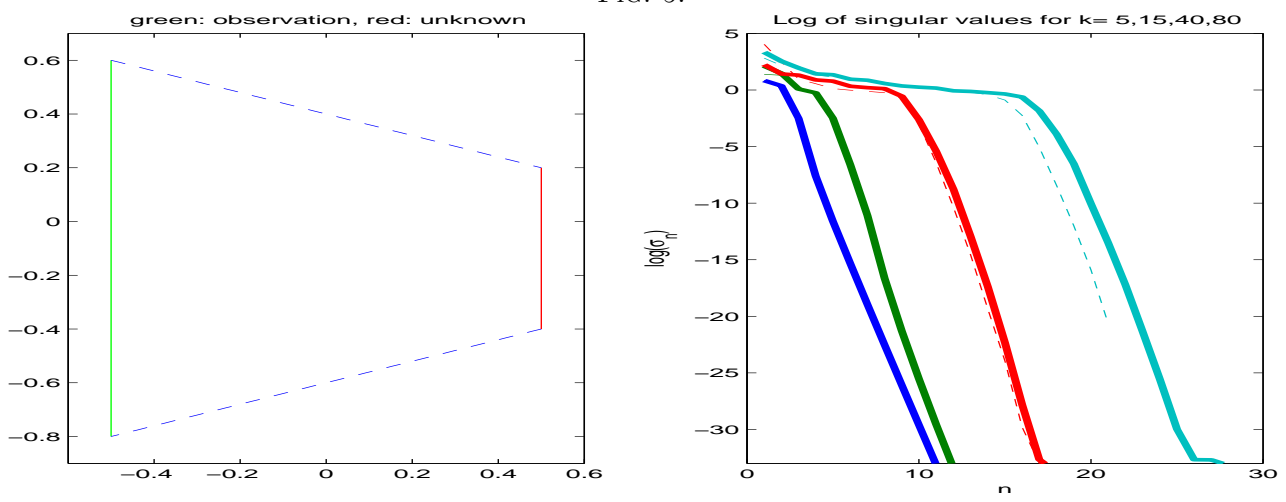

FIG. 6.
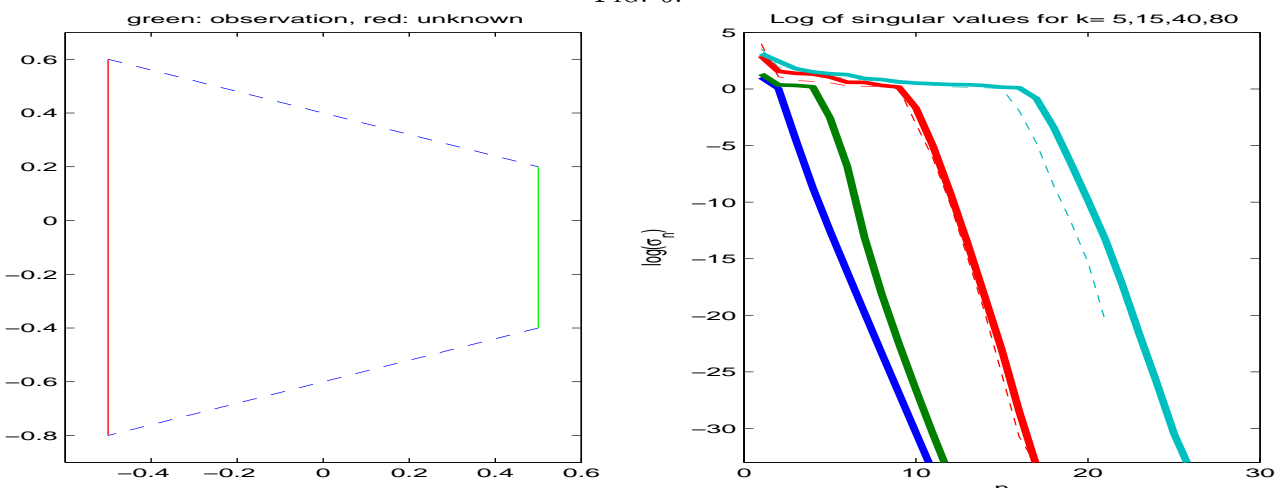

FIG. 7.

wave number for all domains, which is our the main result. The transition curve seems to be approximately linearly increasing with $k$. The slope of the transition point strongly depends on the geometry, where the annulus case is that one with the fastest increase of stability.

The transition point is less pronounced for the annular case (Fig. 8, 9). In order to further validate the numerical computations we also compared the transition curve of Fig. 12 (right), which seemed to be quite ragged with the known analytic solution 

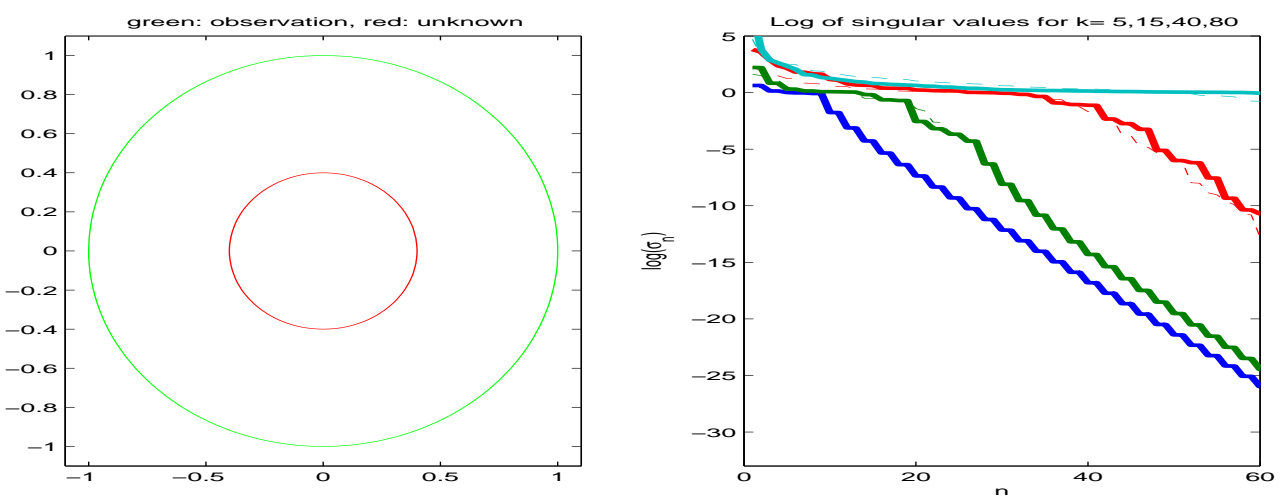

FIG. 8.
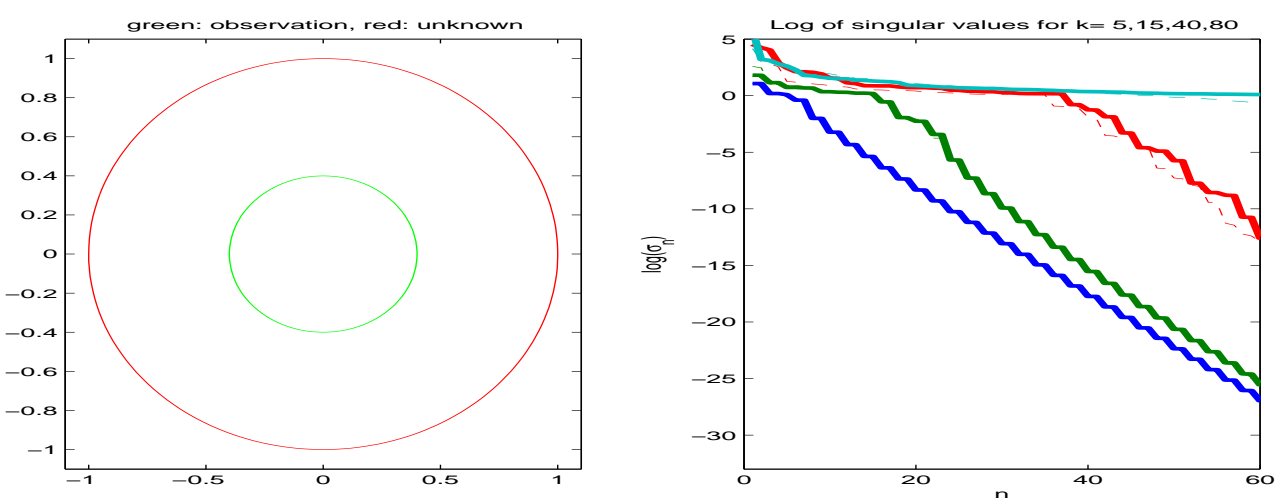

FIG. 9.
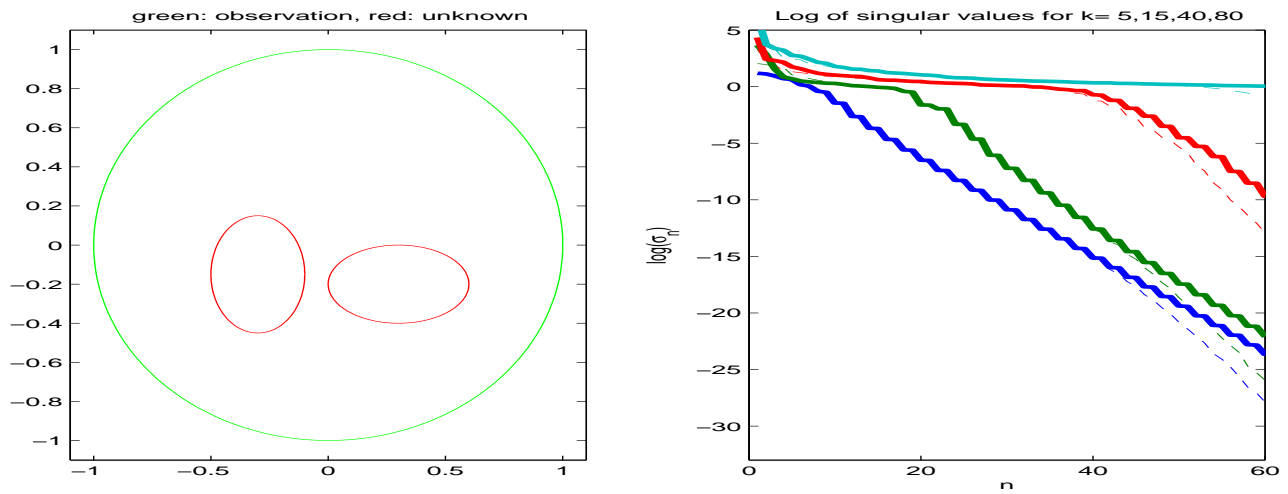

FIG. 10.

where for the latter we used a finer grid on the wave number $k$. The results are plotted in Figure 14. In the left plot the red line corresponds to zero-level set of $\log \left(\sigma_{n}(k)\right)$ using the analytic solution for the annulus case in the geometry of Figure 8 . The blue line corresponds to the numerically computed solution as in Figure 12. The right plot is a computation of the zero-level curve using the analytic formula for higher wave numbers $k$ up to 800 . 

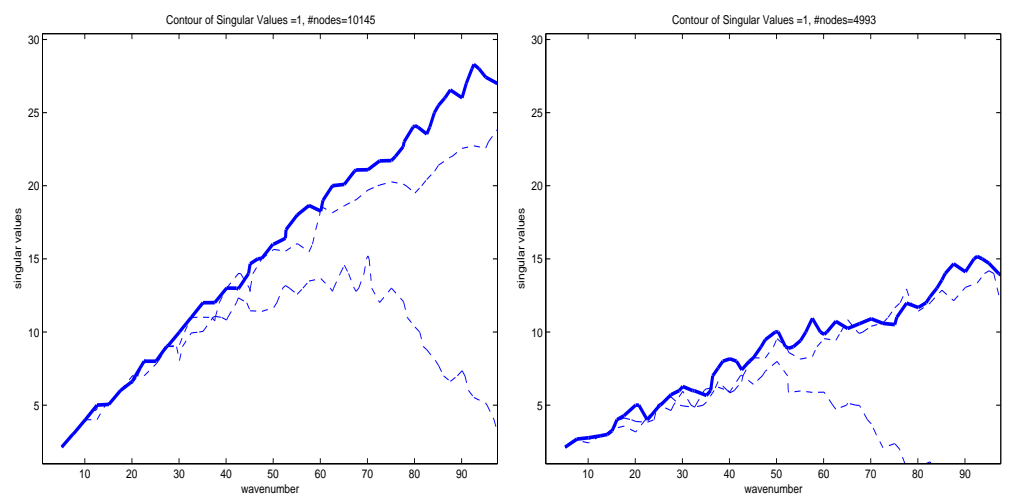

FIG. 11. Zero level curve of $\log \left(\sigma_{n}(k)\right)$, ex. of Fig. 5(left), Fig. 6(right)
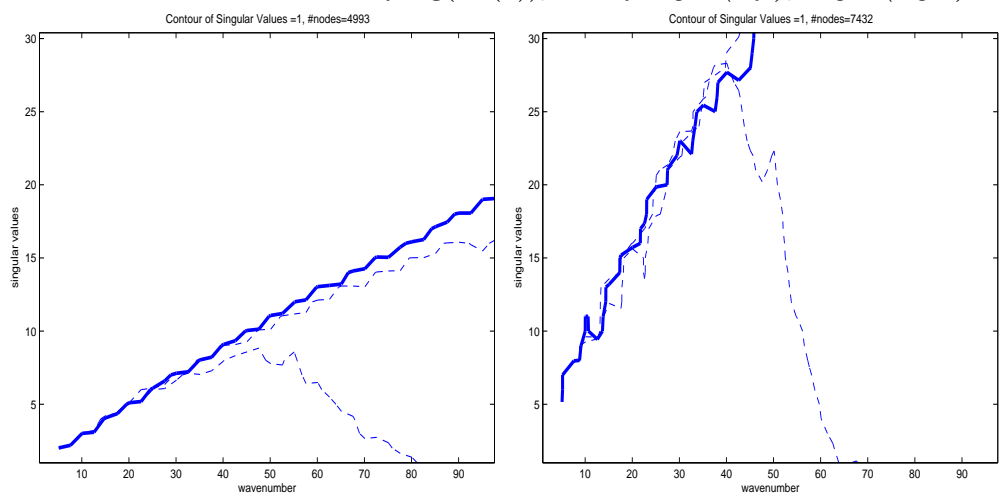

FIG. 12. Zero level curve of $\log \left(\sigma_{n}(k)\right)$, ex. of Fig. 7(left), Fig. 8(right)
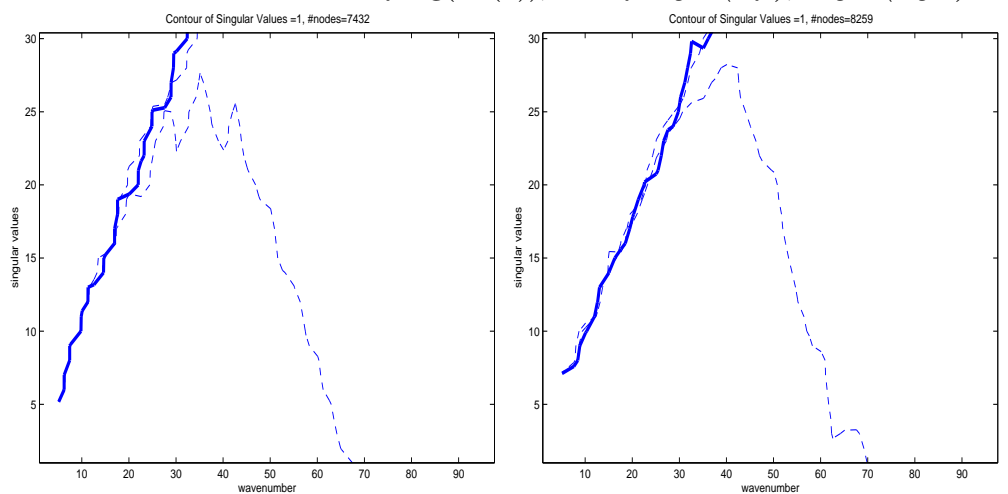

FIG. 13. Zero level curve of $\log \left(\sigma_{n}(k)\right)$, ex. of Fig. 9(left), Fig. 10(right)

Now to the comparison between the analytic and the numerical results: It can be seen that the overall agreement between the two curves is good up to wave number $k \sim 50$, but because of the different resolutions of the computations with respect to the wave number the numerically computed curve does not reveal the fine oscillations that the red line shows. First of all, this indicates that the transition curve is by far a simple one. One might ask further if the numerics can be trusted in view of these high oscillations. Our answer is that this case the zero level curve might not be 

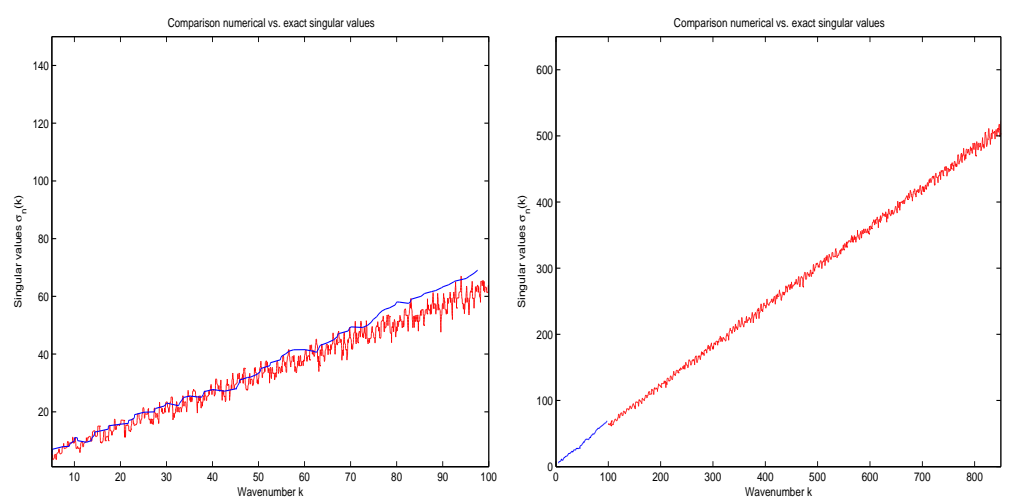

FIG. 14. Comparison of the numerical results of the zero level of $\log \left(\sigma_{n}(k)\right)$ for the geometry of Fig. 8. Left: Red curve: Zero level curve using FEM computation. Blue picture: Zero level using analytic formula. Right: Zero level curve using analytic formula for higher wave number

right point of transition. In fact, plotting a different level curve, e.g., $\log \sigma_{n}(k)=-1$ reveals a much smoother curve. We observed that the transition does not have to be at the point $\sigma_{n}(k)=1$ (which corresponds to the zero level curve of the $\log$ ), but one can take a point $\sigma_{n}(k)=c_{0}$, where $c_{0}$ is a small but fixed constant. Such a constant $c_{0}$ does not alter the situation in the inverse problem, and the analysis of the regularization method can be done in an same way as before. With this modification we observed in all our examples an increasing region of stability. On the left plot we can see that the region of stability is also increasing for higher wave numbers. The figures indicate an almost linear relationship between $k$ and the corresponding transition point $n$. Let us note that even with the analytic formula it was not possible to compute similar results for higher wave numbers $k>1000$, because of rounding errors in the computation of the Bessel function of very high order.

A further computation of the same curve for randomly chosen annuli (i.e. $r_{1}$ and $r_{2}$ are sampled out of a uniform distribution on $\left.[0,1]\right)$ revealed the same picture: an almost linearly increasing function of $k$ of the region of stability. Of course the slope of this function depends on the choice of $r_{1}$ and $r_{2}$.

We observed similar behavior of singular values disregard of trapping properties of $\Omega$ and $\Gamma_{o b s}, \Gamma_{b}$ studied in particular, by Bardos, Lebeau, Rauch [4] for wave equations. When $k$ is growing one can expect that solutions to the Helmholtz equation behave like solutions of the wave equation. But most likely it is true only for "high frequency" solutions, while in a growing subspace of solutions the Cauchy problem is Lipschitz stable. Indeed, Figures 6, 8, 10 correspond to "non trapping geometries", sufficient conditions of exact observability in [4] are satisfied, and it is not a surprise that stability in the Cauchy problem for the corresponding Helmholtz equation is increasing. In more detail, in Figure 6 for any point of $\Omega$ and any direction one of a brocken straight lines starting at this point and in this or opposite direction after reflections from $\Gamma_{b}$ will intersect $\Gamma_{o b s}$. However, Figures 7, 9 correspond to trapping geometries, resulting in exponential instability of "high frequency" solutions as in [17]. In Figure 7, the line parallel to the red interval after reflections will intersect green interval where there is no data. So this line can viewed as a trapped one. But nevertheless, somehow surprisingly, there seems to be the same subspace of Lipschitz stable solutions which is increasing with $k$. 

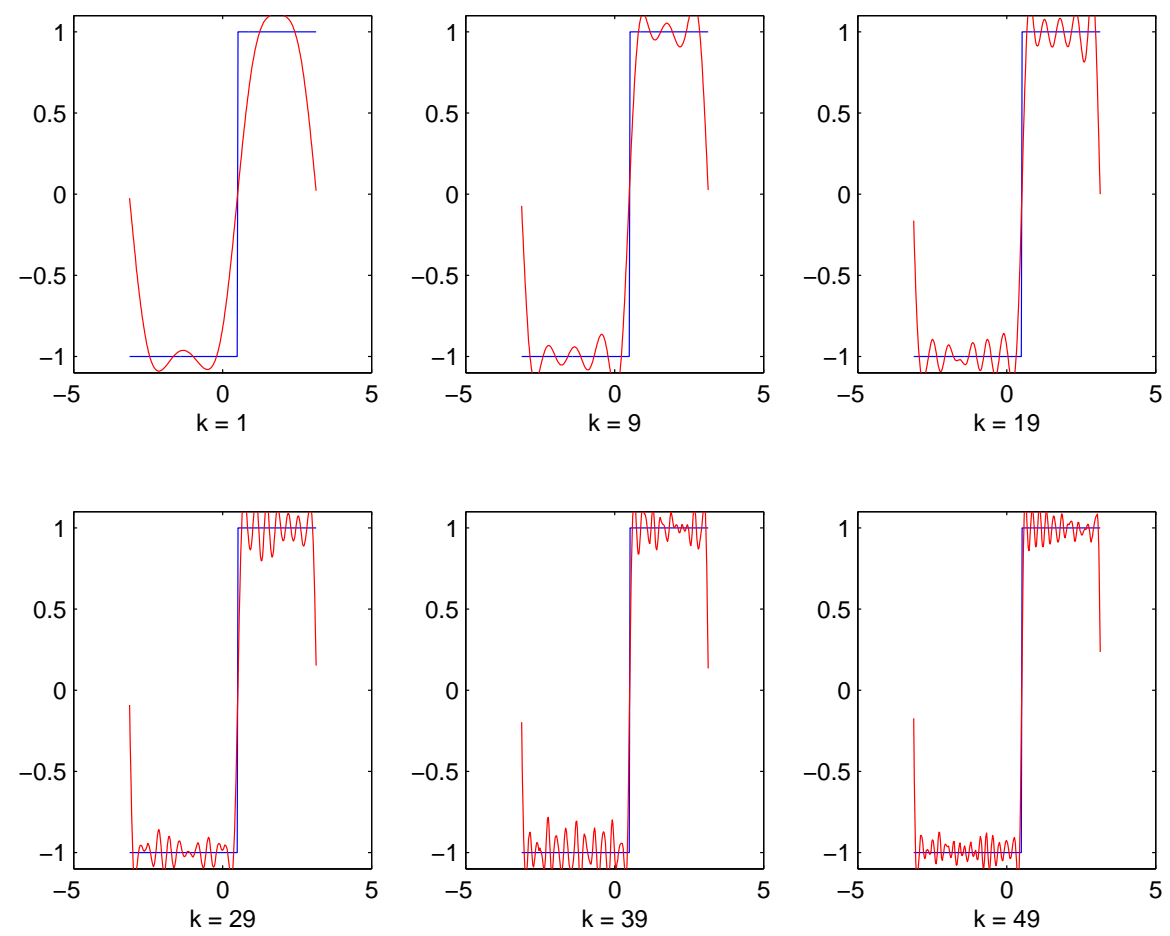

FIG. 15. Regularized solution and exact solution for fixed $\alpha$ and different wave numbers $k$

These observations let us formulate a conjecture: For an arbitrary domain, the Assumption 1 is satisfied.

5. Numerical solution of the inverse problem. In this section we apply Tikhonov regularization to the Cauchy problem in the form (13) and compare the convergence predicted by Theorems 1, 3, 4 with the numerical ones. We fix an annular domain with observation on the interior radius and unknown on the outside as in Fig. 9. As exact solution $x^{\dagger}$ we choose a) a sign function and b) a Gaussian curve:

$$
x_{1}^{\dagger}=\operatorname{sign}(\phi-0.5), \quad x_{2}^{\dagger}=e^{-\frac{\phi^{2}}{0.5^{2}}}, \quad \phi \in[-\pi, \pi],
$$

where $\phi$ is the angle on the outer circle. The first function is a nonsmooth one, hence we can expect at most logarithmic rates in this case. The second function is smooth with exponentially decaying Fourier coefficients, hence this is a case where a Hölder source condition holds.

At first we want to verify the increased stability indicated by (30), (31). Note that the estimates in these equations improve with increasing $k$ for fixed $\alpha$. We hence computed a regularized solution for $x_{1}^{\dagger}$ and $x_{2}^{\dagger}$ for a fixed regularization parameter and fixed noise level but for different wave number. The results can be seen in Figures 15, 16. Figure 15 shows the case of the discontinuous exact solution $x_{1}^{\dagger}$. The regularization parameter was chosen as $\alpha=0.01$, the noise level was $1 \%$ additive Gaussian noise. Figure 16 displays the case of the smooth exact solution $x_{2}^{\dagger}$. What can be seen is, that the approximation improves with increasing $k$, which clearly indicates that the problem becomes more stable. Note that we used a $L^{2}$-penalization, so the oscillations in Fig. 15 are due to Gibbs phenomenon. In Fig. 16 the results seem to 

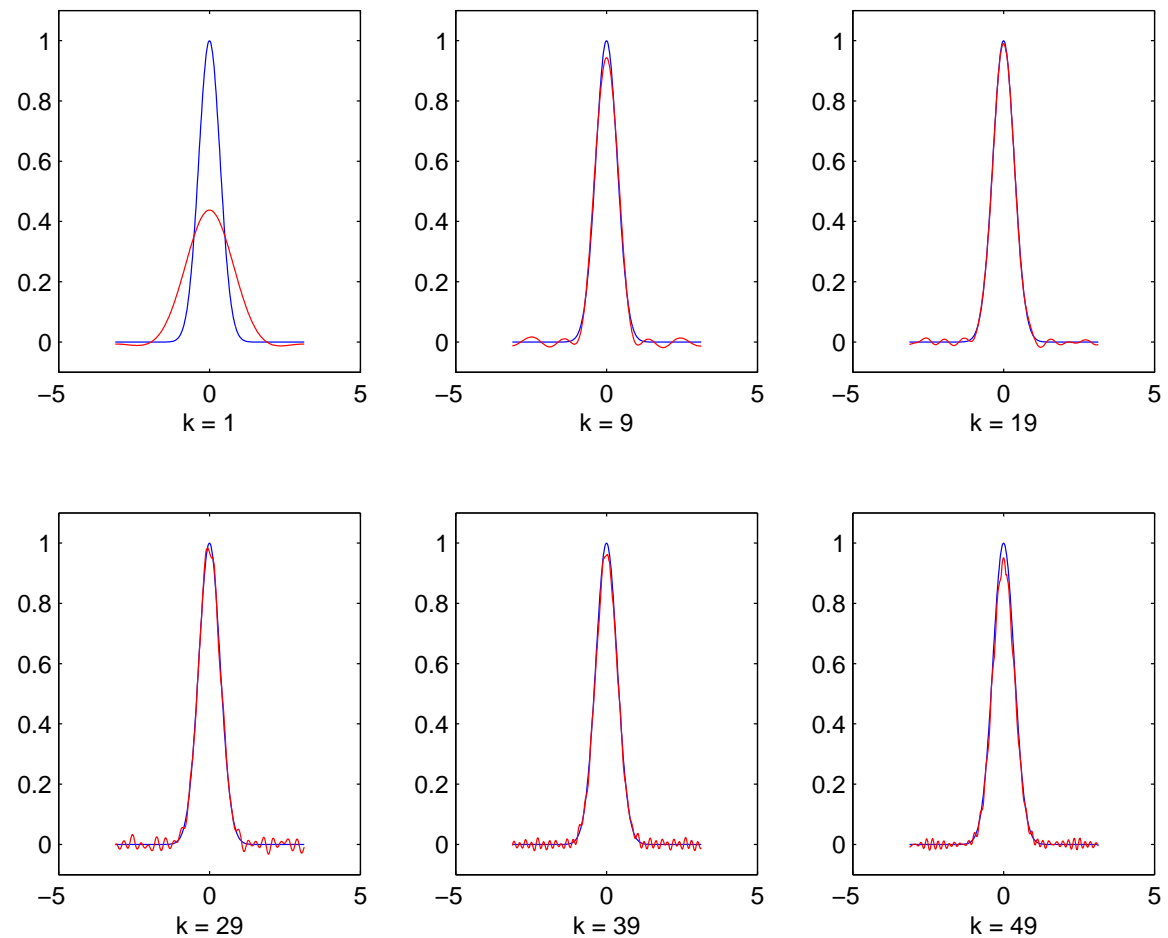

FIG. 16. Regularized solution and exact solution for fixed $\alpha$ and different wave numbers $k$
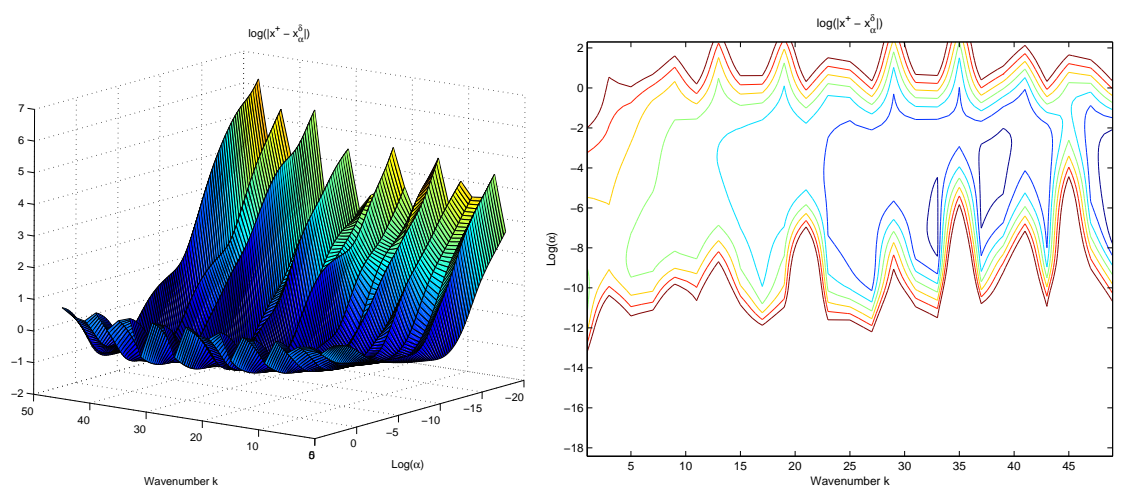

FIG. 17. Log of the error for different $\alpha$ and $k$, noise level fixed $\delta=1 \%$

deteriorate at the peak slightly in the case $k=39$ and $k=49$, for which we do not have an clear explanation.

The next Figure 17 shows a plot of the logarithm of the error between the exact solution and the regularized solution for different regularization parameter and different wave number for fixed noise level $\delta=1 \%$ and for the exact solution $x_{1}^{\dagger}$. The right plot is a surface plot and the left one a contour plot. In the figures it can be seen that for fixed $k$ the grows for small $\alpha$ and for large $\alpha$ with a minimum in between. This is totally consistent with the general theory of regularization, where for small $\alpha$ the propagated data noise dominates and for large $\alpha$ the approximation error dominates. 

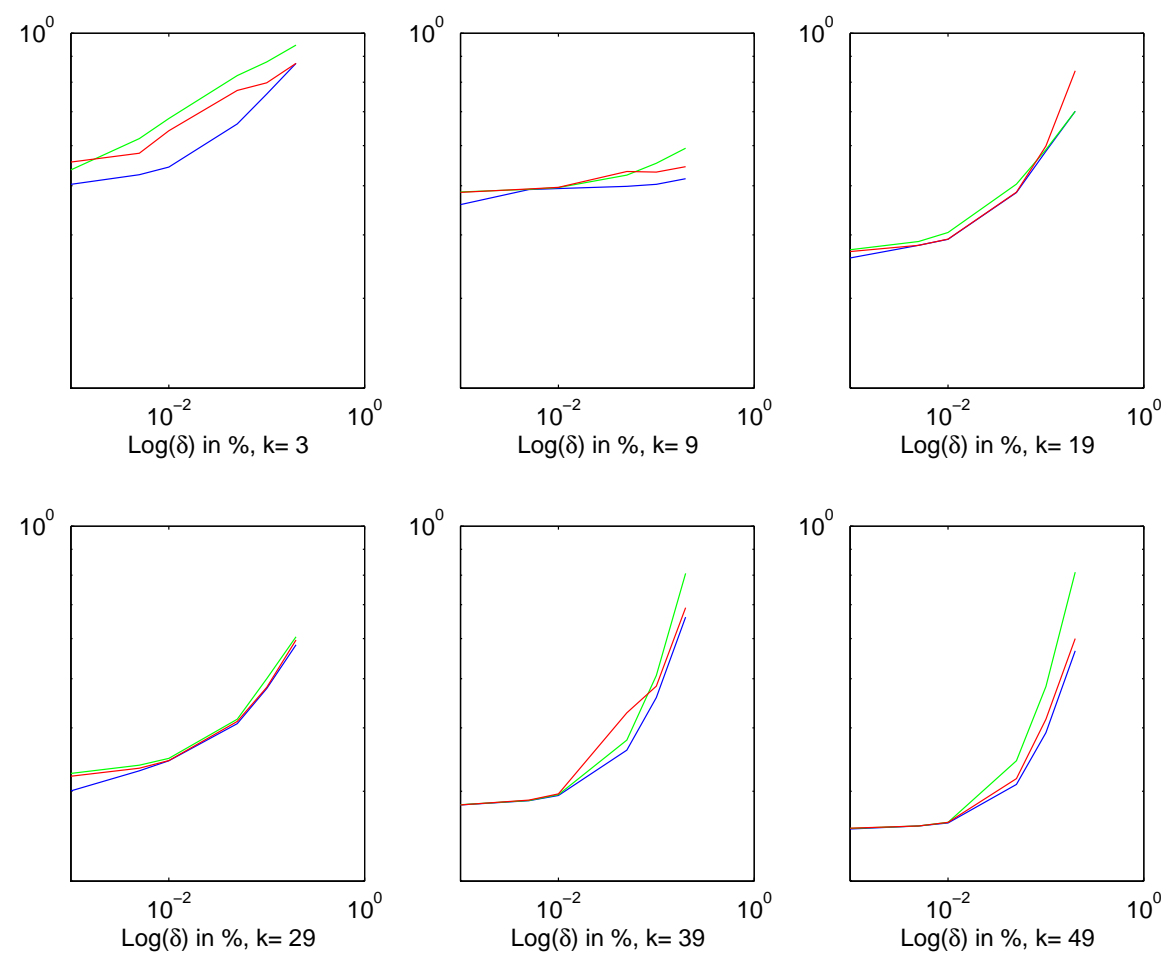

FIG. 18. Log of the error for different noise levels and for different parameter choice rules and different wave numbers

What is important with respect to the $k$-dependence is that the minimal error (over $\alpha$ ) decreases with $k$. In fact, for $k=5$ the minimal error that can be achieved for this noise level is about 5 times larger than the minimal error achievable for $k \sim 50$. This again indicates the increasing stability.

The final plots compare different parameter choice rules for different noise levels and wave number. We choose $\alpha$ according to two parameter choice rules, the discrepancy principle, i.e. the largest $\alpha$ such that the residual is smaller than $1.1 \delta$, and the quasi-optimality principle. We compared this with the optimal choice, i.e. with the results for choosing $\alpha$ such that the error $\left\|x_{\alpha}^{\delta}-x_{1}^{\dagger}\right\|$. The results are shown in Figure 18. In the plot the abscissa corresponds to the logarithm of the noise level in percent, the ordinate corresponds to the $\log$ of the error $\left\|x_{\alpha}^{\delta}-x_{1}^{\dagger}\right\|$. The blue line corresponds to the optimal parameter, the green line to that for the discrepancy principle and the red one for the quasi-optimality principle. We observed that the quasi-optimality is in most cases better than the discrepancy principle, which often chooses a too large regularization parameter. In fact the quasi-optimality principle gave a smaller error in $75 \%$ of the cases. It can be seen from the figures that with increasing wave number the error becomes uniformly smaller, consistent with the increased stability. What we also observed is not only a smaller total error for higher wave number, but the parameter choice of $\alpha$ becomes less significant. In fact, for higher wave number there is a broader range from which the regularization parameter can be chosen, still giving good result. In the plots this can be seen from the fact that the three curves are closer together for higher $k$ than for $k=3$. Finally, let us note 
that all the computations for the regularization were performed with Gaussian noise. Our main intention was to indicate the improvement of the results with increasing $k$. We therefore used a noise that is uniform and does not depend on $k$.

6. Conclusion. We investigated the singular value distribution of the forward operator associated to the Cauchy problem for the Helmholtz equation. As a main result we found a sequence of subspaces increasing with the wave number $k$ on which the problem is well-posed. The numerical computations indicate that this subspace of stability appears for all domains (at least in the two-dimensional case), in particular also for those that do not satisfy convexity conditions. The presence of the subspace of stability for arbitrary domains, still lacks a satisfactory theoretical explanation. Postulating the existence of such a subspace of stability we analyzed the error for Tikhonov regularization. We obtained asymptotically linear rates. Important for this asymptotic rate is the use of the projected noise level (not the usual noise level) and the corresponding parameter choice rule. Our analysis was done for Tikhonov regularization but there is no problem to extend this to other regularization methods as well. We finally gave numerical evidence, that the error between the exact and regularized solution is smaller for higher wave numbers, hence, the problem shows an increasing stability (although it is of course still ill-posed). A numerical evidence of increasing stability in inverse problems can be found in [3, 7, 21, 22]. Finally, we think that a similar analysis can be done and is useful for other problems in partial differential equations, like the lateral Cauchy problem and inverse problems for parabolic and hyperbolic equations and systems. This will be our research goal for near future.

Acknowledgement. The work of Victor Isakov was supported in part by the Emylou Keith and Betty Dutcher Distinguished Professorship and the NSF grants DMS 07-07734 and DMS 10-08902. A part of this research was conducted during the Mini Special Semester on Inverse Problems, May 18-July 15, 2009 in Linz, organized by RICAM, Austrian Academy of Sciences.

\section{REFERENCES}

[1] G. Alessandrini, L. Rondi, E. Rosset, And S. Vessella, The stability for the cauchy problem for elliptic equations, Inverse Problems, 25 (2009), p. 123004 (47pp).

[2] I. M. BABušKa AND S. A. SAUter, Is the pollution effect of the FEM avoidable for the Helmholtz equation considering high wave numbers?, SIAM J. Numer. Anal., 34 (1997), pp. 23922423.

[3] G. BAO AND P. LI, Inverse medium scattering problem for the Helmholtz equation at a fixed frequency, Inverse Probl., 21 (2005), pp. 1621-1641.

[4] C. Bardos, G. Lebeau, And J. Rauch, Sharp sufficient conditions for the observation, control, and stabilization of waves from the boundary, SIAM J. Control Optimization, 30 (1992), pp. 1024-1065.

[5] Y. Chen, On the shielding effect of the Helmholtz equation., Commun. Pure Appl. Math., 61 (2008), pp. 627-638.

[6] T. Delillo, V. Isakov, N. Valdivia, and L. Wang, The detection of surface vibrations from interior acoustic pressure, Inverse Problems, 19 (2003), pp. 507-524.

[7] M. Eller And N. P. VAldivia, Acoustic source identification using multiple frequency information, Inverse Problems, 25 (2009), p. 115005 (20pp).

[8] H. W. Engl, M. Hanke, And A. Neubauer, Regularization of inverse problems., Kluwer, Dordrecht, 1996.

[9] J. HADAmARD, Lectures on Cauchy's problem in linear partial differential equations, Yale University Press, New Haven, 1923.

[10] B. Hofmann And S. Kindermann, On the degree of Ill-posedness for linear problems with non-compact operators, to appear in Methods of Applications and Analysis, (17) (2011). 
[11] B. Hofmann, P.Mathe, And M.Schieck, Modulus of continuity for conditionally stable illposed problems in hilbert space., J. Inv. Ill-posed Problems, (2008), pp. 567-585.

[12] T. Hohage, Logarithmic convergence rates of the iteratively regularized Gauss-Newton method for an inverse potential and an inverse scattering problem., Inverse Probl., 13 (1997), pp. 1279-1299.

[13] - Regularization of exponentially ill-posed problems., Numer. Funct. Anal. Optimization, 21 (2000), pp. 439-464.

[14] T. HRYCAK AND V. ISAKOV, Increased stability in the continuation of solutions to the Helmholtz equation., Inverse Probl., 20 (2004), pp. 697-712.

[15] V. IsAKOV, Increased stability in the continuation for the Helmholtz equation with variable coefficient. Ancona, Fabio (ed.) et al., Control methods in PDE-dynamical systems. AMSIMS-SIAM joint summer research conference, Snowbird, UT, USA, July 3-7, 2005. Providence, RI: American Mathematical Society (AMS). Contemporary Mathematics 426, 255267 (2007)., 2007.

[16] I. IsLOMOV, On construction of a regularized solution of the Cauchy problem for the Helmholtz equation., Dokl. Akad. Nauk UzSSR, 1984 (1984), pp. 10-11.

[17] F. John, Continuous dependence on data for solutions of partial differential equations with a prescribed bound., Commun. Pure Appl. Math., 13 (1960), pp. 551-585.

[18] S. Kindermann And A. Neubauer, On the convergence of the quasioptimality criterion for (iterated) Tikhonov regularization., Inverse Probl. Imaging, 2 (2008), pp. 291-299.

[19] L. Marin, L. Elliott, P. J. Heggs, D. B. Ingham, D. Lesnic, and X. Wen, Conjugate gradient-boundary element solution to the Cauchy problem for Helmholtz-type equations., Comput. Mech., 31 (2003), pp. 367-377.

[20] - Comparison of regularization methods for solving the Cauchy problem associated with the Helmholtz equation., Int. J. Numer. Methods Eng., 60 (2004), pp. 1933-1947.

[21] F. Natterer and F. WüBbeling, A propagation-backpropagation method for ultrasound tomography., Inverse Probl., 11 (1995), pp. 1225-1232.

[22] - Marching schemes for inverse acoustic scattering problems., Numer. Math., 100 (2005), pp. $697-710$.

[23] H. QIn, T. WEI, AND R. SHI, Modified Tikhonov regularization method for the Cauchy problem of the Helmholtz equation., J. Comput. Appl. Math., 224 (2009), pp. 39-53.

[24] T. Regińska And K. Regiński, Approximate solution of a Cauchy problem for the Helmholtz equation., Inverse Probl., 22 (2006), pp. 975-989.

[25] D. Aralumallige Subbarayappa and V. Isakov, On increased stability in the continuation of the Helmholtz equation, Inverse Probl., 23 (2007), pp. 1689-1697.

[26] D. Aralumallige Subbarayappa and V. Isakov, Increasing stability of the continuation for the Maxwell system, Inverse Probl., 26 (2010), 074005.

[27] E. G. Williams, Regularization methods for near-field acoustical holography., J. Acoust. Soc. Am., 110 (2001), pp. 1976-1988.

[28] X.-T. XIONG AND C.-L. FU, Two approximate methods of a Cauchy problem for the Helmholtz equation., Comput. Appl. Math., 26 (2007), pp. 285-307. 
V. ISAKOV AND S. KINDERMANN 